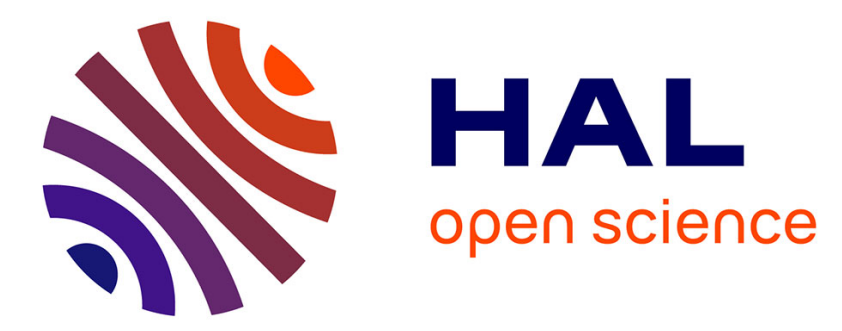

\title{
Path Filters: A Class Of True In-Line Topologies With Transmission Zeros
}

Stefano Tamiazzo, Giuseppe Macchiarella, Fabien Seyfert

\section{To cite this version:}

Stefano Tamiazzo, Giuseppe Macchiarella, Fabien Seyfert. Path Filters: A Class Of True In-Line Topologies With Transmission Zeros. IEEE Transactions on Microwave Theory and Techniques, 2022, 70 (1), pp.850 - 863. 10.1109/TMTT.2021.3126861 . hal-03449926

\section{HAL Id: hal-03449926 \\ https://hal.inria.fr/hal-03449926}

Submitted on 25 Nov 2021

HAL is a multi-disciplinary open access archive for the deposit and dissemination of scientific research documents, whether they are published or not. The documents may come from teaching and research institutions in France or abroad, or from public or private research centers.
L'archive ouverte pluridisciplinaire HAL, est destinée au dépôt et à la diffusion de documents scientifiques de niveau recherche, publiés ou non, émanant des établissements d'enseignement et de recherche français ou étrangers, des laboratoires publics ou privés. 


\title{
Path Filters: A Class of True In-Line Topologies With Transmission Zeros
}

\author{
Stefano Tamiazzo, Giuseppe Macchiarella, Fellow, IEEE, and Fabien Seyfert
}

\begin{abstract}
In this paper we present a comprehensive discussion of a new class of inline microwave filters with transmission zeros in the response, namely the path filters. The main features of this filters class are highlighted, and an original (synthesis-based) design approach is presented, relying on the derivation of suitable characteristic polynomials. In addition to the classical Generalized Chebyshev characteristic, two new characteristics are introduced (namely the Bounded Chebyshev and the Reduced Chebyshev), that allow improving the flexibility in the requirements assignment of path filters. A new method for the synthesis of the lowpass prototype is also introduced, that overcome the limitation in the classical synthesis based on the manipulation of the transversal prototype (whose synthesis may fail in case of path filters). Finally, the proposed approach for designing the class of considered filters has been validated by several examples that include the evaluation of the characteristic polynomials, the prototype synthesis and the dimensioning of the physical structures in waveguide technology.
\end{abstract}

Index Terms - Characteristic polynomials, In-line filters, Synthesis, Transmission zeros.

\section{INTRODUCTION}

$\mathrm{T}$ HE development of latest generation telecommunication equipment is pushing towards implementing solutions close to the limits of current technologies. For example, filters used in terminals and radio units for $5 \mathrm{G}$, as well as those used for satellite communications, are required to be more and more selective, with low passband losses and strictly constrained in terms of overall volume and weight. For this reason, in recent years, in addition to developing new and more performing materials and fabrication technologies, new conceptual solutions concerning the filter configurations are being studied [1]-[13]. As well known, the inline configuration composed by direct-coupled cavities is the simplest and most compact filter topology, widely used for decades [1]. It however suffers from scarce selectivity because no transmission zeros are allowed in the response. For this reason, many efforts have been done during the years to introduce transmission zeros in the response of inline filters without losing the advantages of this convenient topology. Extracted-pole configurations were among the first solutions adopted [2]-[6], although they do not allow for a true inline topology, require rejection cavities and are not easy to

Manuscript received June 7, 2021; revised October 7, 2021; Accepted October 12, 2021.

This article is an expanded version from the IEEE MTT-S International Microwave Symposium (IMS 2020), Atlanta, GA, USA, June 20-25, 2021. (Corresponding author: Giuseppe Macchiarella) design. More effective solutions are represented by inline filters with frequency-dependent couplings [7]-[13]. These filters feature true inline topology but requires special coupling structures that must provide both the required coupling in the passband and complete rejection at the transmission zero frequency. Recently, ingenious solutions for quasi-inline filters in coaxial technologies have been proposed, based on the even and odd modes of strongly coupled resonators [14]-[15]. Finally, in [16] a true inline waveguide filter with transmission zeros has been realized by introducing a cross coupling between non-adjacent cavities by means of a wire running through the cavities.

All the above-mentioned solutions present some drawbacks due to either the not true inline configuration or to the requirement of special components, or for the complex design techniques required (not within the reach of on average skilled designers).

This work is focused on a new category of true inline filters recently proposed, namely the path filters [17]-[18]. The main advantage of this type of inline filter is that its implementation does not require special components. Moreover, the sign of the couplings does not affect the magnitude of the response (as it happens in all-pole filters). In practice, the practical dimensioning of path filters is carried out exactly in the same manner of direct-coupled all-pole filters. On the other hand, the design of path filters exhibiting the Generalized Chebyshev characteristic with assigned transmission zeros is allowed only for a specific set of transmission zeros and the passband return loss (RL) cannot be assigned freely [18]. The first proposed approach to the design of path filters is based on the optimization of the coupling matrix [17]. More recently, a method for the analytical synthesis of symmetric path filters is presented in [18]. In the present work we introduce a general approach to the design of path filters, removing the symmetry constraint and broadening the set of assignable transmission zeros. The paper is organized as follows. In Section II the path filters topology is recalled and the constraints concerning its polynomial characterization are derived. Section III introduces the evaluation of "ad-hoc" polynomials models for path filters. In Section IV we discuss a suitable synthesis technique for the prototype network based on the derived polynomials (in fact,

S. Tamiazzo is with Commscope, Via Archimede, Agrate Brianza (Mi) - Italy (e-mail: stefano.tamiazzo@commscope.com). G. Macchiarella is with Dipartimento di Elettronica, Informazione e Bioingneria, Politecnico di Milano, 20133 Milano, Italy (e-mail: giuseppe.macchiarella@polimi.it). Fabien Seyfert is with INRIA, 06902 Sophia-Antipolis, France (e-mail: fabien.seyfert@inria.fr). 
usual synthesis techniques may fail when applied to these polynomials). Some examples of path filters design in waveguide technology are then illustrated in Section V while Section VI concludes the paper.

\section{DEFINITIONS AND POLYNOMIAL ChARACTERIZATION}

The basic topology of path filters (originally introduced in [18]) is shown in Fig. 1. It is comprised of $n p$ resonant nodes cascaded along a straight "path", which also includes the input (source) and output (load) nodes. Source (S) and load (L) separate the $n p$ resonators into three blocks. The first block, comprised of $n^{S}$ nodes, can be seen as "hanging" from the source and will be referred to as the source dangling branch of order $n^{S}$. The middle block, comprised of $n^{D}$ nodes, will be referred to as the mainline path filter. The third block, comprised of $n^{L}$ nodes, can be seen as "hanging" from the load and will be referred to as the load dangling branch of order $n^{L}$. Either the source or the load dangling branch, but not both, might be missing, in which case we will set $n^{S}=0$ or $n^{L}=0$.

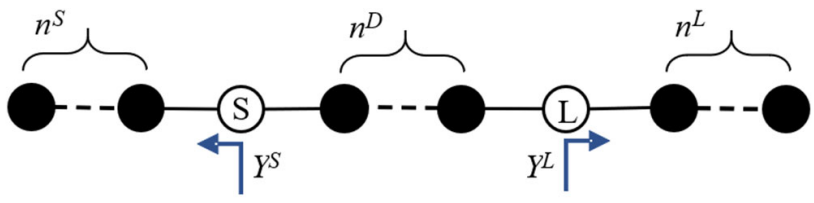

Fig. 1. General configuration of path filters. The black circles represen either a resonator (bandpass equivalent circuit) or a unit capacitance in parallel with a frequency-invariant susceptance (normalized lowpass prototype). The lines are the coupling inverters, and the white nodes are the source and load, possibly with an added frequency invariant susceptance in parallel (NRN)

A circuital representation of the generic path filter is shown in Fig. 2a, where $Y^{S}$ and $Y^{L}$ (defined in Fig. 1) represent the source and load dangling admittances, whereas $S^{D}$ is the scattering matrix of the mainline filter.

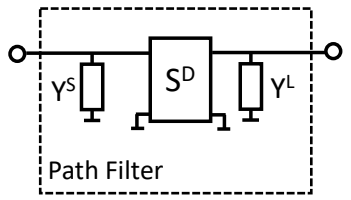

a)

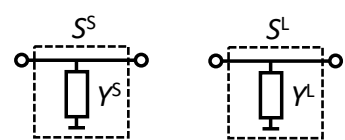

b)
Fig. 2. a) Compact representation of path filters. b) Two-port corresponding to the dangling branches at Source and Load

The circuit element $Y^{S}$ is a lossless positive real (p.r.) one-port admittance function, whose distinct poles $j \Omega_{z, k}^{S}$, with $k=1, \ldots, n^{S}$, are necessarily simple and purely imaginary (p.r. property) with positive real residues $\left\{r_{k}^{s}\right\}$. Thus, the admittance function $Y^{S}$ admits the following representation:

$$
Y^{S}(s)=\frac{q^{S}(s)}{m^{S}(s)}=j a^{S}+\sum_{k=1}^{n^{s}} \frac{r_{k}^{S}}{s-j \Omega_{z, k}^{S}}
$$

where $a^{S}$ is a real number; $q^{S}(s)$ and $m^{S}(s)$ are polynomials whose order can differ at most by one. This leads to the polynomial representations of the matrix $S^{S}$ in (2) characterizing the two-port built on the source dangling branch, as depicted in Fig. 2b.

$$
S^{S}=\frac{1}{2+Y^{S}}\left[\begin{array}{cc}
-Y^{S} & 2 \\
2 & -Y^{S}
\end{array}\right]=\frac{1}{2 m^{S}+q^{S}}\left[\begin{array}{cc}
-q^{S} & 2 m^{S} \\
2 m^{S} & -q^{S}
\end{array}\right]
$$

Similar considerations hold true for the admittance function $Y^{L}$, with poles $\left\{j \Omega_{z, k}^{L}\right\}$, and for the matrix $S^{L}$ of Fig. $2 \mathrm{~b}$.

The overall scattering matrix $S$ of a path filter can be computed in terms of the three $2 \times 2$ scattering matrices $S^{S}, S^{D}$ and $S^{L}$ as follows:

$$
S=S^{S} \odot S^{D} \odot S^{L}
$$

where the symbol $\odot$ is the cascade operator for two 2-port $S$ matrices, with port 2 of the first network connected to port 1 of the second network.

We assume in the following the apex $T$ as either $S$ or $L$. At the $n^{T}$ frequencies $j \Omega_{z, k}^{T}$ the corresponding dangling branch is shorted and thus the $n^{T}$ poles $j \Omega_{z, k}^{T}$ of $Y^{T}$ are also poles of the input and output admittances $\left(Y_{\text {in/out }}\right)$ of the filter:

$$
Y_{\text {in/out }}\left(j \Omega_{z, k}^{T}\right)=\infty \quad\left(k=1, \ldots, n^{T}\right)
$$

These imply that:

$$
S_{11 / 22}\left(j \Omega_{z, k}^{T}\right)=-1, \quad S_{12}\left(j \Omega_{z, k}^{T}\right)=0, \quad\left(k=1, \ldots, n^{T}\right)
$$

In conclusion, the poles of the admittances $Y^{S}$ and $Y^{L}$ are exactly the $n_{z}=n^{S}+n^{L}$ transmission zeros (TZs) of the path filter. Those from $Y^{S}\left(j \Omega_{z, k}^{S}\right)$ are said to be generated at the source, while those from $Y^{L}\left(j \Omega_{z, k}^{L}\right)$ are said to be generated at the load. The minimum path rule [19] assures that no other finite $\mathrm{TZ}$ other than these can exist in path filters.

Although $\Omega_{z, k}^{S}$ and $\Omega_{z, k}^{L}$ are distinct for each branch, we might have repetition of some zeros between the two branches, i.e., $\Omega_{z, k}^{S}=\Omega_{z, h}^{L}=\Omega_{z}$ for some values of the indices $k$ and $h$. In this case $j \Omega_{z}$ (the TZ) will be generated at both branches and therefore represent a double $\mathrm{TZ}$ of the overall response $\left(S_{12}\left(j \Omega_{z}\right)=\partial S_{12} /\left.\partial \Omega\right|_{\Omega=\Omega_{z}}=0\right.$ must hold $)$.

Path filters have the minimal number of coupling elements and do not require additional couplings for producing TZs; they are "true" inline configuration. Moreover, unlike other similar topologies (e.g., those using frequency-dependent couplings [7]-[13]), no special implementation for coupling elements or resonators is required and all couplings can be made positive if so desired.

Although $n^{S}$ and $n^{L}$ are in principle arbitrary, dangling branches generating two or more transmission zeros with the same sign (that is, on the same side of the passband) result, for the alternating singularities property of $Y^{S}$ and $Y^{L}$, in at least one zero of $Y^{S}$ (or $Y^{L}$ ) resting in between a pair of adjacent poles, or TZs of the filter, often causing a significant degradation of the stopband attenuation, especially when the two TZs are close to each other. We will then limit the maximum number of TZs per dangling branch to two or less, with no dangling branch generating a pair of zeros with the same sign, or on the same side of the passband. 
The design of lossless path filters starts, as usual, with the synthesis of a prototype with a desired response. The prototype is defined in the normalized lowpass domain $s=j \Omega$, which is related to the bandpass domain $(f)$ by the usual frequency transformation $\Omega=\left(f / f_{0}-f_{0} / f\right) / B_{n}$ with $f_{0}$ passband center frequency and $B_{n}$ normalized bandwidth.

The synthesis of the lowpass prototype requires first the evaluation of the characteristic polynomials $F(s), P(s)$ and $E(s)$ in the complex normalized frequency variable $s=j \Omega$, which define the scattering parameters of the filter:

$$
S_{11}=\frac{F(s)}{E(s)}, \quad S_{22}=\frac{(-1)^{n p} F(s)^{*}}{E(s)}, \quad S_{21}=\frac{P(s)}{E(s)}
$$

where $\left({ }^{*}\right)$ denotes the para-conjugate operator [20]. $E(s)$ is a strictly Hurwitz polynomial and its roots are the poles of the filter, while the roots of $F(s)$ and $P(s)$ are the reflection zeros (RZs) and the transmission zeros (TZs) respectively.

In addition to the usual lossless-ness constraints, path filters have the additional constraints (4), which, in terms of the characteristic polynomials in (5), are expressed as

$$
\begin{array}{ll}
E\left(j \Omega_{z, k}^{S}\right)=-F\left(j \Omega_{z, k}^{S}\right), & k=1, \ldots, n^{S} \\
E\left(j \Omega_{z, k}^{L}\right)=-(-1)^{n p} F\left(j \Omega_{z, k}^{L}\right)^{*}, & k=1, \ldots, n^{L}
\end{array}
$$

The characteristic polynomials $E(s)$ and $F(s)$ of path filters must then satisfy the $n^{S}+n^{L}$ conditions expressed by (6). Conversely, if equations (6) are satisfied for each of the $n_{z} \mathrm{TZs}$ of a filter, then this filter admits a path realization, as will be proved in section IV.

\section{A. Polynomials evaluation (Generalized Chebyshev characteristic)}

In this work we will consider several classes of single-band, non-fully canonical equiripple path filter responses, that is equiripple responses for which conditions (4), or equivalently (6), can be enforced. The first class is Generalized Chebyshev (GC) path filters, for which all RZs are purely imaginary, and relations (5) specialize as shown in (5a) (the subscript 0 indicates reference to GC filters):

$$
S_{110}=S_{220}=\frac{F_{0}(s)}{E_{0}(s)}, \quad S_{210}=\frac{j^{\left(n^{D}+1\right)} p_{0} P_{0}(s)}{E_{0}(s)}
$$

where $E_{0}(s)$ and $F_{0}(s)$ are monic polynomials of degree $n p$ and $P_{0}(s)$ is also a monic polynomial of degree $n_{z}$. The real constant $p_{0}$ is related to the in-band return loss level (RL) by:

$$
p_{0}^{2}=\left|\frac{F_{0}(j)}{P_{0}(j)}\right|^{2}\left(10^{R L / 10}-1\right)
$$

To have an idea of why conditions (4) are achievable for certain GC filters with a single TZ, consider the plot in Fig. 3. It is related to a family of GC filters of order $n p=3$, with one $\mathrm{TZ}$ at $j \Omega_{z}=1.1 j$ and various values of RL levels in the range from 1 to $60 \mathrm{~dB}$.

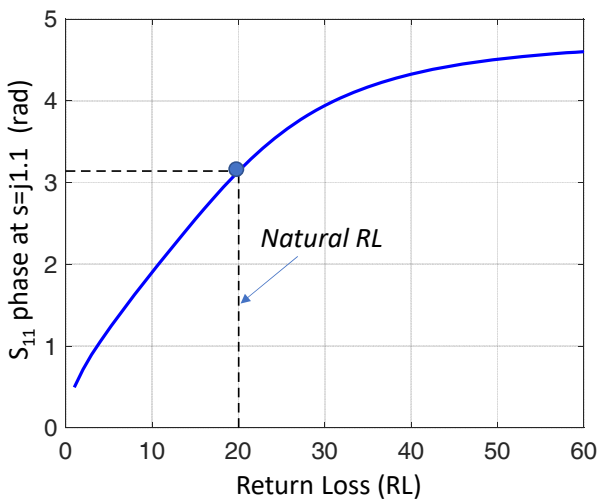

Fig. 3. Unwrapped phase of $\mathrm{S}_{11}$ at the $\mathrm{TZ}=j 1.1$ frequency for several $3^{\text {rd }}$ degree Chebyshev filter of various RL level.

For each RL point the corresponding value of the unwrapped phase of $S_{11}$ at $s=j 1.1$ has been plotted. It is observed that the unwrapped phase is an increasing and continuous function of the RL. When the return loss goes to zero $\left(\mathrm{S}_{11, \max }=1\right)$ the phase goes to zero, while for larger and larger RL values the phase goes to a limit which is greater than $\pi$. The continuity of the curve assures that a RL level exists such that $\operatorname{Arg}\left(S_{11}(j 1.1)\right)=$ $\pi$, or $S_{11}(j 1.1)=-1$ (in Fig. 3 this RL level is equal to 20.0237 $\mathrm{dB})$. Setting the RL to this value will then guarantee that conditions (4) are met and that a realization in the form of a path filter exists. More generally, for any fixed value $\Omega_{z}$ of the TZ and any filter order $n p \geq 3$, we get at least one value of RL, called in the following the natural $R L$ level relative to $\left|\Omega_{z}\right|$ and $n p$, such that $\operatorname{Arg}\left(S_{11}\left(j \Omega_{z}\right)\right)=(2 k+1) \pi$ for some integer $k$. For higher degrees it is possible that there are several solutions of the RL satisfying this condition, but in practice only the lowest one is usable in most applications.

To compute the natural RL level relative to $\left|\Omega_{z}\right|$ and $n p$ of a GC filter with a single $\mathrm{TZ}$ at $j \Omega_{z}$ the following algorithm can be used:

1. Compute $P_{0}(s)$

2. Evaluate the RZs determining the Chebyshev characteristic and compute $F(s)$. Note that this step is independent of the RL level of the filter.

3. Choose (if first iteration) or update RL level and compute $p_{0}$ with (7)

4. Evaluate the $E(s)$ by spectral factorization:

$$
E_{0}(s) E_{0}(s)^{*}=F_{0}(s) F_{0}(s)^{*}+p_{0}^{2} P_{0}(s) P_{0}(s)^{*}
$$

5. Check constraints (6)

6. Not satisfied: go back to step 3

7. Satisfied: End of the procedure

The update of RL at step 3 must be carried out so as the error $e=\left|E_{0}\left(j \Omega_{z}\right)+F_{0}\left(j \Omega_{z}\right)\right|^{2}$ is minimized. In practice, the procedure consists in the numerical solution of equation $e=0$ with $R L$ as the unknown. In addition to the case of single $\mathrm{TZ}$, this procedure can be extended to GC path filters with other admissible TZ patterns, with various combinations of values for $n_{z}, n^{S}$ and $n^{L}$. Four of these patterns, all characterized by a single real number $\Omega_{z}$, are as follows: 
1) A single arbitrary $T Z$ :

$$
n^{S}=1 \text { and } \Omega_{z}^{S}=\left\{\Omega_{z}\right\} \text { or } n^{L}=1 \text { and } \Omega_{z}^{L}=\left\{\Omega_{z}\right\}
$$

2) A pair of symmetric TZs:

$n^{S}=2$ and $\Omega_{z}^{S}=\left\{-\Omega_{z}, \Omega_{z}\right\}$ or $n^{L}=2$ and $\Omega_{z}^{L}=\left\{-\Omega_{z}, \Omega_{z}\right\}$

3) A pair of coincident TZs:

$$
n^{S}=n^{L}=1, \Omega_{z}^{S}=\left\{\Omega_{z}\right\}, \quad \Omega_{z}^{L}=\left\{\Omega_{z}\right\}
$$

4)Two coincident pairs of symmetric TZs:

$$
n_{z}=4, n^{S}=n^{L}=2, \quad \Omega_{z}^{S}=\left\{-\Omega_{z}, \Omega_{z}\right\}, \quad \Omega_{z}^{L}=\left\{-\Omega_{z}, \Omega_{z}\right\}
$$

As an example, consider the case $n p=8$ and $\Omega_{z}=1.1$.
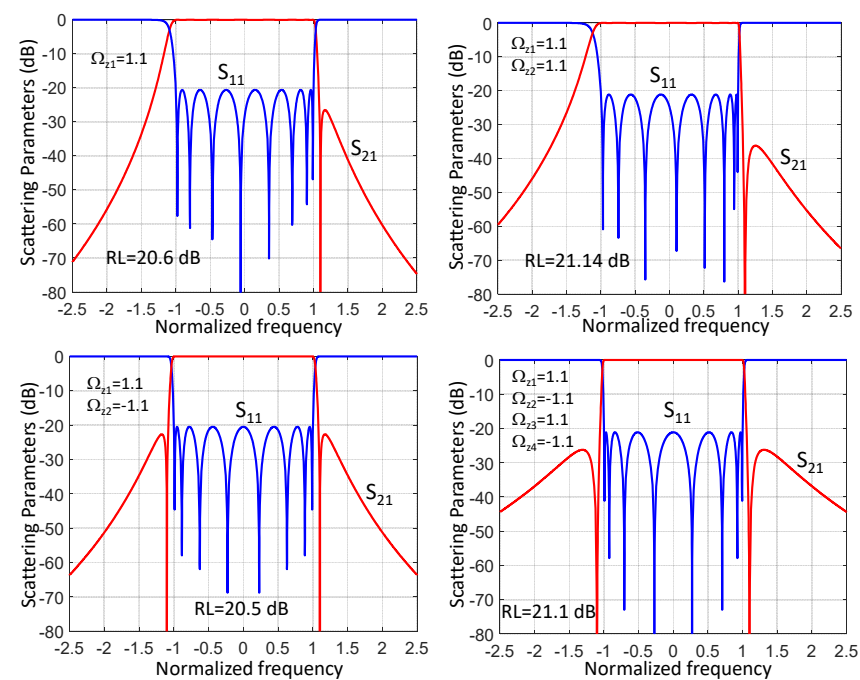

Fig. 4. Response from the computed polynomials for the four possible patterns of TZs. The $R L$ value obtained in each case is also shown

Fig. 4 shows the scattering parameters $S_{110}$ and $S_{210}$ computed from the evaluated polynomials for the four patterns of TZs above specified. The corresponding natural $R L$ value, slightly different in each case, is also shown. In all these cases, the reflection zeros are imaginary so, from (5), $S_{110}=S_{220}$. Thus, the condition assumed in [18] for the synthesis of path filters is fulfilled and the same results found in [18] can be obtained with the procedure here introduced, which is much simpler to implement numerically than the one proposed in [18].

\section{B. Synthesis flexibility increased by NRNs at source/load}

The main drawback of the so realized path filters is the lack of flexibility in assigning the TZs. To begin with, the natural RL may become very large when the TZs are distant from the passband. In fact, the natural RL is an increasing function of $\left|\Omega_{z}\right|$ for fixed $n p$. Fig.5 shows the case of a single transmission zero for three values of $n p$. In all cases, when $\left|\Omega_{z}\right|$ is approximately greater than 1.3 , RL becomes excessively large, thus penalizing the attenuation in the stopbands. This limitation will be addressed in section III.

Secondly, when $n_{z}>1$, the set of admissible TZs is required to exhibit certain symmetries, according to one of the four patterns listed above. To overcome the latter limitation, and gain more freedom in the polynomial modeling of path filters, it is possible to specialize eq. (5a) by introducing two phase terms $\theta_{1}$ and $\theta_{2}$ in the scattering polynomials definition as follows:

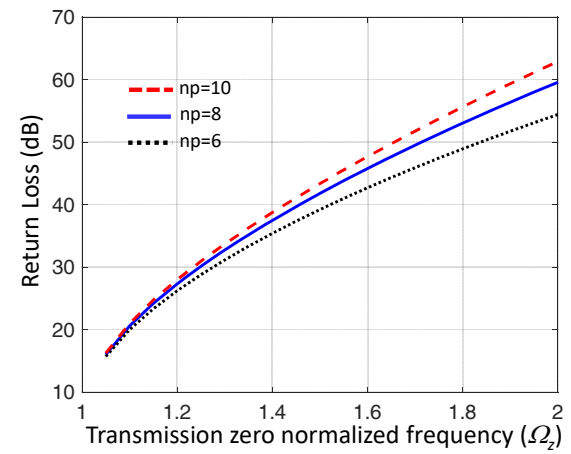

Fig. 5. Natural RL vs. normalized transmission zero frequency for three values of filter order and single TZ.

$$
\begin{aligned}
& S_{11}=e^{j\left(\theta_{1}\right)} S_{110}(s), \\
& S_{22}=e^{j\left(\theta_{2}\right)} S_{220}(s), \\
& S_{21}=e^{j\left(\theta_{1}+\theta_{2}\right) / 2} S_{210}(s)
\end{aligned}
$$

It can be easily verified that the scattering parameters in (8) satisfy the lossless requirements. Moreover, unlike $S_{110}$ and $S_{220}$ which are equal with the GC characteristic, $S_{11}$ and $S_{22}$ can now be different. From the circuital realization point of view, the addition of a phase term in $S_{11}$ and $S_{22}$ requires two nonresonating nodes (NRN) implemented with susceptances in parallel to the source and load, whose value is given by $b_{S}=-\tan \left(\theta_{1} / 2\right)$ and $b_{L}=-\tan \left(\theta_{2} / 2\right)$ [4]. We can then extend the class of path filters shown in Fig. 1 by assuming that a non-zero frequency-invariant susceptance might exist in parallel to the unit resistor representing source and load.

By introducing these phase terms, the class of equiripple frequency response for path filters can be extended. Consider first the problem of realizing a GC response with two arbitrary $\mathrm{TZs}\left(j \Omega_{z}^{S}, j \Omega_{z}^{L}\right)$ and an arbitrary RL level, by means of a path filter with $n^{S}=n^{L}=1$. The scattering parameters in (8) are defined by the polynomials $F_{0}(s), E_{0}(s)$ and $p_{0} P_{0}(s)$, and the angles $\theta_{1}$ and $\theta_{2}$. Imposing the constraints (4") in (8), being $S_{220}=S_{110}$, we get:

$$
\begin{aligned}
& S_{110}\left(j \Omega_{z}^{S}\right) e^{j \theta_{1}}=-1 \Rightarrow \phi_{110}\left(j \Omega_{z}^{S}\right)+\theta_{1}= \pm \pi \\
& S_{110}\left(j \Omega_{z}^{L}\right) e^{j \theta_{2}}=-1 \Rightarrow \phi_{110}\left(j \Omega_{z}^{L}\right)+\theta_{2}= \pm \pi
\end{aligned}
$$

where $\phi_{110}\left(j \Omega_{z}^{S}\right)$ and $\phi_{110}\left(j \Omega_{z}^{L}\right)$ are the phases of $S_{110}$ at the two TZs frequencies. From (9) the expression for $\theta_{1}$ and $\theta_{2}$ can be derived:

$$
\begin{aligned}
& \theta_{1}=-\phi_{110}\left(j \Omega_{z}^{S}\right) \pm \pi \\
& \theta_{2}=-\phi_{110}\left(j \Omega_{z}^{L}\right) \pm \pi
\end{aligned}
$$

The introduction of the phase terms in (8) therefore allows to implement the prescribed GC characteristic with the desired $R L$ and also have the path condition satisfied.

Observing the synthesis result in the considered case $\left(n^{S}=n^{L}=\right.$ 1 ) it is however easy to verify that the synthesized path filter is 
equivalent to an extracted-pole configuration [20], for which there are well-known synthesis techniques [4] that allow to arbitrarily assign the return loss for any pair of TZs.

A path filter configuration, not equivalent to the extracted-pole one, is instead obtained when the two TZs are extracted by the same dangling branch, for example in the case $\left(n^{S}=2, n^{L}=0\right)$. If we call $j \Omega_{z, 1}^{S}$ and $j \Omega_{z, 2}^{S}$ the two transmission zeros realized by the branch of order 2 at the input, then the new interpolation condition we get from (4) takes following form:

$$
S_{110}\left(j \Omega_{z, 1}^{S}\right)=S_{110}\left(j \Omega_{z, 2}^{S}\right) .
$$

As a consequence, the two transmission zeros should be of opposite signs. Eventually the phase constants are given from (9) by:

$$
\begin{aligned}
& \phi_{110}\left(j \Omega_{z, 1}^{S}\right)=\phi_{110}\left(j \Omega_{z, 2}^{S}\right) \\
& \theta_{1}=-\phi_{110}\left(j \Omega_{z, 1}^{S}\right) \pm \pi=-\phi_{110}\left(j \Omega_{z, 2}^{S}\right) \pm \pi
\end{aligned}
$$

The first condition in (11) can be satisfied only for a certain value(s) of $p_{0}$, determining the admissible (natural) $R L$ level(s), while with the second condition we find $\theta_{1}$ (and then the susceptance $b_{S}$ in parallel to the source representing the NRN).

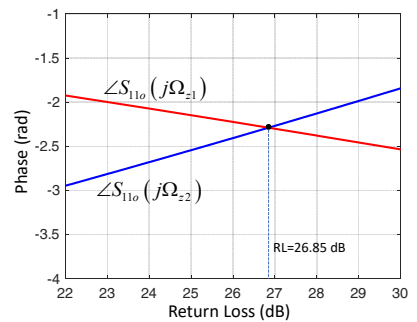

(a)

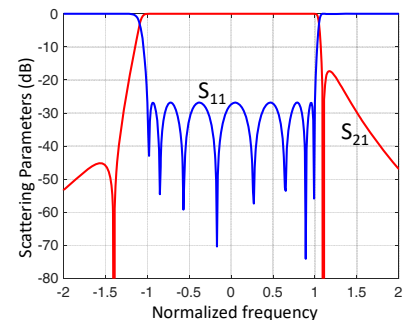

(b)
Fig. 6. (a): Phase of $S_{110}$ at $\Omega_{z 1}$ and $\Omega_{z 2}$ vs. $R L$ ( $E_{0}, F_{0}, P_{0}$ computed for Chebyshev response with $n p, R L$ and TZs assigned). (b): Computed polynomials response $\left(n p=8, \Omega_{z}^{S}=[-1.41 .1], R L=26.85 \mathrm{~dB}\right)$.

Although we still cannot assign the RL arbitrarily, the inclusion of a NRN within a second order dangling branch allows to introduce a pair of asymmetric TZs of opposite sign in the frequency response of a path filter.

As an example, consider $n p=8$ and $\Omega_{z}^{S}=[-1.4,1.1]$. Fig. 6a shows $\phi_{110}\left(j \Omega_{z, 1}^{S}\right)$ and $\phi_{110}\left(j \Omega_{z, 2}^{S}\right)$ vs. RL. It can be observed that the two angles are equal for $\mathrm{RL}=26.85 \mathrm{~dB}$. Using the second equation in (10) we get $\theta_{l}=-0.8521 \mathrm{rad}$ (then $\left.b_{S}=0.454\right)$. The response computed from $E(s), F(s)$ and $P(s)$ polynomials is shown in Fig. $6 \mathrm{~b}$.

In the case of three asymmetric TZs of mixed sign, by adding a NRN at both source and load, we can assign two TZs at opposite sides of the passband to the source dangling branch and the remaining $\mathrm{TZ}$ to the load dangling branch. Consider as an example $n p=8, \Omega_{z}^{S}=[-1.25,1.1], \Omega_{z}^{L}=1.2$. The natural $R L$ solution is found by solving the first condition in (11) imposed at the two TZs assigned to the source dangling branch $\left(\phi_{110}(-j 1.25)=\phi_{110}(j 1.1)\right)$. We obtain $\mathrm{RL}=24.78 \mathrm{~dB}$. Then we get from (9) $\theta_{1}=-0.4838 \mathrm{rad}$ and $\theta_{2}=0.3404 \mathrm{rad}$ (the NRN susceptances result $b_{S}=0.247, b_{L}=-0.172$ ).

The response computed from the characteristic polynomials is shown in Fig. 7a.
With four TZs, there is only one combination of zeros that is allowed: $\Omega_{z}^{S}=\left[\Omega_{z, 1},-\Omega_{z, 2}\right], \Omega_{z}^{L}=\left[-\Omega_{z, 1}, \Omega_{z, 2}\right]$. In fact, the conditions $\phi_{110}\left(j \Omega_{z, 1}\right)=\phi_{110}\left(-j \Omega_{z, 2}\right)$ and $\phi_{110}\left(-j \Omega_{z, 1}\right)=\phi_{110}\left(j \Omega_{z, 2}\right)$ can be simultaneously satisfied with a single value of RL due to the symmetry of the response about the zero frequency. From (10) $\theta_{1}$ and $\theta_{2}$ can then be derived. Fig. 7b shows the response with $\Omega_{z, 1}=1.1, \Omega_{z, 2}=1.225$ and $n p=8$ (for which $\mathrm{RL}=24.62 \mathrm{~dB}$, $\theta_{1}=-\theta_{2}=-0.483 \mathrm{rad}$ and $b_{S}=-b_{L}=0.246$ )

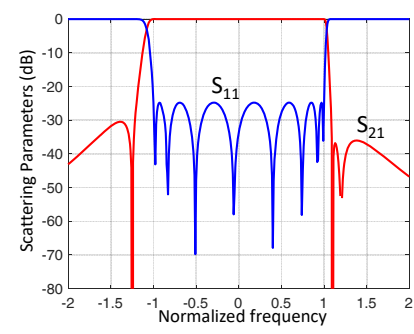

(a)

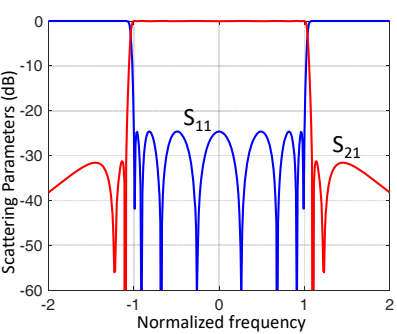

(b)
Fig. 7. (a) Response of the filter with $n p=8, \Omega_{z S}=[-1.5,1.1], \Omega_{z L}=[1.2]$, $R L=24.78 \mathrm{~dB}, \theta_{2}=0 \mathrm{rad}, \theta_{1}=-0.852 \mathrm{rad}$. (b). Response of the filter with $n p=8$, $z S=[-1.225 \mathrm{i}, 1.1 \mathrm{i}], z L=[-1.1 \mathrm{i}, 1.225 \mathrm{i}], \mathrm{RL}=24.62 \mathrm{~dB}, \theta_{2}=0.483 \mathrm{rad}, \theta_{l}=-0.483$ $\mathrm{rad}$.

We have seen above that the introduction of NRNs has increased the flexibility in assigning the transmission zeros of path filters; but the RL cannot yet be arbitrarily assigned. Furthermore, the practical implementation of a NRN in parallel (or series) to the source or load can increase the filter complexity and distort the response due to the frequency dependence of the real structures implementing the NRN (a short- or open- circuited stub). For this reason, new solutions have been devised to allow flexible transmission zeros and RL assignment without increasing the complexity in the practical implementation of path filters.

Up to now we have considered only quasi-elliptic responses with no reflection zeros in the right half-plane: the phase of $S_{11}$ and $S_{22}$ is therefore entirely determined from the knowledge of their modulus (up to a constant if NRNs are used). When we vary the RL level in search for a natural solution, we change the magnitude and therefore the phase variation in the stopband of $\mathrm{S}$ parameters, where specific phase values at the TZs frequencies are required. But there are still limits to what can be achieved with this technique, even when NRNs are contemplated. In fact, the RL level cannot be set arbitrarily and symmetry constraints in the location of the TZs when $n_{z}=4$ are still present, as the discussion above has put in evidence.

To remove these limits, the assumption of imaginary RZs needs to be removed.

\section{Path Filters With Assignable RetURn Loss}

We have seen in Section II that GC path filters do not allow to freely assign the return loss, not even when NRNs are used. On the other hand, it would be desirable to adjust the RL level of path filters to recover at least some of the stopband attenuation degradation that the often-large natural RL values are associated to. One way to accomplish this goal is to remove the assumption of purely imaginary RZs while preserving the 
equiripple property of $S_{11}$. In doing so, one can follow either of two complementary strategies:

- Prescribing a small but non-zero real part to all reflection zeros.

- Prescribing a relatively large real part to one or two reflection zeros while keeping the remaining zeros on the imaginary axis.

More specifically, when the desired RL level is not too far from a natural solution, a slight move of all RZs (that will become complex) might suffice to adjust the phase winding of $S_{11}$ and/or $S_{22}$ along the segments of the imaginary axis comprising the two stopbands as required. Conversely, when distant from a natural solution, one needs something like one or two all pass factor(s), that does not change the magnitude, but again accelerates the phase winding of $S_{11}$ and/or $S_{22}$ along the two stopbands. This can be obtained by deliberately moving one or two RZs away from the imaginary axis, depending on the number and location of the TZs, as will be clearer in the following.

Accordingly, we have then devised two non-Chebyshev, but equiripple, polynomial models.

\section{A. Path Filters with the Bounded Chebyshev Characteristic}

The first polynomial model we propose relies on a new characteristic function $C_{n}^{\prime}(\Omega)$ having an equiripple response in the passband with maxima and minima at about the same frequencies as the GC characteristic function $C_{n}(\Omega)$. However, the minima of $C_{n}^{\prime}(\Omega)$ are not zero, so that the frequencies where the minima occur are no longer the reflection zeros (the latter becoming complex frequencies). In Appendix $\mathrm{A}$ the derivation of the $C_{n}^{\prime}(\Omega)$ is described, together with the evaluation of the characteristic polynomials $E, F$ and $P$.

In addition to the order $n p$ and the transmission zeros $j \Omega_{z}$, the new characteristic function (referred to as Bounded Chebyshev) requires two key parameters: the minimum $\left(R L_{\min }\right)$ and maximum $\left(R L_{\max }\right)$ return loss in the passband. There is then an additional degree of freedom that can be exploited when modeling path filters polynomials. This means that, in case of one TZ, we can set $R L_{\min }$ and look for the $R L_{\max }$ determining characteristic polynomials satisfying conditions (6) (the same procedure illustrated in Section IIA can be used). It is found that $R L_{\text {min }}$ can be set to values even larger than the natural $R L$. In case of two symmetric TZs, we can assign $R L_{\text {min }}$ freely whereas, for two asymmetric zeros, one or more fixed (not RL adjustable) solutions $\left(R L_{\min }, R L_{\max }\right)$ may exist, depending on $n p$ and the assigned TZs, with $R L_{\min }$ and $R L_{\max }$ getting closer and closer as the asymmetry of the TZs increases. No solution exists for 3 and 4 TZs unless specific symmetries occur. The Bounded Chebyshev function has also another interesting feature: the signs of the real part of the reflection zeros does not affect the magnitude of $S$ parameters (only the phase is modified). This means that there are $2^{n p}$ different $F(s)$ polynomials (with fixed $P$ and $E$ ), that may produce different results when conditions (6) are checked. For instance, in case of $1 \mathrm{TZ}$, the solution with $R L_{\text {min }}$ smaller than the natural $R L$ tends to have RZs with positive real parts whereas the opposite is true for $R L_{\text {min }}$ larger than the natural $R L$. For a given level of $R L_{m i n}$, one may find several Bounded Chebyshev realizations with different sequences of signs of the real part of the reflection zeros and different $R L_{\max }$ levels accordingly. Among these, one should choose the one with higher $R L_{\max }$ which in turn will provide the greatest stopband rejection. In case of two asymmetric TZs, different solutions (i.e., pairs of $R L_{\min }, R L_{\max }$ ) can be found for specific sequence of signs.

For additional flexibility, NRNs can be introduced also with the bounded Chebyshev characteristic. In particular, NRNs can deal with the TZs asymmetry whereas $R L_{\max }$ can be determined for any prescribed value of $R L_{\text {min }}$.

Regarding the evaluation of the polynomials defining the path filters response when more than one $\mathrm{TZ}$ is assigned we want to point out that this consists in the solution of a non-linear system (derived from the constraints (6)), dependent on two or more unknowns. The solution of this system is generally achieved by optimization-based numerical methods (in the examples shown in this work we have used the function $f$ solve of Matlab ${ }^{\circledR}$ optimization toolbox).

As a first example, let consider $n p=7$ and $\Omega_{z}^{S}=[-1.1,1.2]$. The solution with the Bounded Chebyshev characteristic is found only for specific sequences of sign of the real part of the reflection zeros $(z F)$. For instance, one of these is given by: $z F=[-0.014-0.9911 \mathrm{i},-0.0595-0.8624 \mathrm{i},-0.1147-0.5262 \mathrm{i}$, $0.139-0.0263 \mathrm{i}, 0.1177+0.479 \mathrm{i}, 0.0662+0.8341 \mathrm{i}$, $0.0183+0.9879 \mathrm{i}]$.

The resulting return loss bounds are $R L_{\min }=19.97 \mathrm{~dB}, R L_{\max }=$ $23.31 \mathrm{~dB}$. Introducing a NRN on the source, we can assign $R L_{\min }=20 \mathrm{~dB}$ and obtain a solution with all positive signs of the reflection zeros, $R L_{\max }=29.35 \mathrm{~dB}$, and $\theta_{1}=0.3863 \mathrm{rad}$ $\left(b_{S}=-0.1956\right)$

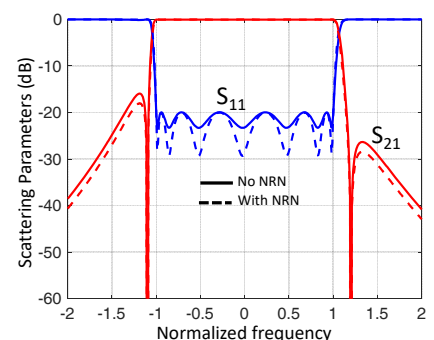

(a)

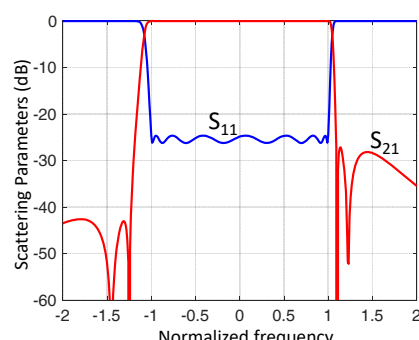

(b)
Fig. 8. (a) Response of the filter with $n p=7, \Omega_{z S}=[-1.1,1.2]$. Solid lines: no NRN at source. Dashed lines: NRN at source. (b) Response of the filter with $n p=8, \Omega_{z S}=[-1.25,1.1], \Omega_{z L}=[-1.45,1.225]$. NRNs are used at source and load.

Fig. 8a shows the $\mathrm{S}$ parameters in the two cases. It can be noticed that the addition of the NRN on the source provides a larger attenuation in the stopbands, for the same value of $R L_{\min }$.

The second example assumes $n p=8$ and $\Omega_{z}^{S}=[-1.25,1.1]$, $\Omega_{z}^{L}=[-1.45,1.225]$. In this case no solution is possible without NRNs, whose introduction allows several solutions for different sequence of signs of the real part of the reflection zeros. One of these sequences $\quad(z F=[-0.0194-0.995 \mathrm{i}, \quad-0.0685-0.8855 \mathrm{i}$, $-0.1341-0.6213 \mathrm{i}, \quad-0.1849-0.1872 \mathrm{i}, \quad 0.178+0.3143 \mathrm{i}$, $-0.1158+0.713 \mathrm{i}, \quad-0.05+0.9238 \mathrm{i}, \quad 0.01225+0.997 \mathrm{i}])$ gives 
$R L_{\min }=24.63 \mathrm{~dB}, R L_{\max }=26.23 \mathrm{~dB}, \theta_{1}=-0.6722 \mathrm{rad}, \theta_{2}=-0.3768$ $\mathrm{rad}\left(b_{S}=0.349, b_{L}=0.191\right)$. The response obtained by the computed polynomials is shown in Fig. 8b.

\section{B. Path Filters with Reduced Chebyshev Characteristic}

Although the Bounded Chebyshev characteristic has further increased the degrees of freedom in assigning RL and transmission zeros, constraints still exists in these assignments, especially when a marked asymmetry exists in the desired response and the use of NRNs is not desired. We have then devised a second way to realize a non-Chebyshev equiripple response of degree $n p$ where the polynomial $F(s)$ possesses $n c$ complex zeros (with $n c=1$ or 2 ) and the remaining $n p-n c$ zeros are purely imaginary. It can be observed that the characteristic function underlying this response has $n p-n c$ maxima and minima in the passband. Moreover, the attenuation it produces in the stopbands falls somewhere in between that obtained with the GC characteristics of order $n p-n c$ and $n p$. We refer to this characteristic as the Reduced Chebyshev characteristic whose detailed evaluation is described in Appendix B.

The Reduced Chebyshev characteristic makes available up to four free parameters in the synthesis of path filters, that can be exploited to satisfy the constraints (6) while preserving the equiripple response in the passband. In this way, without using NRNs, no constraints are required on the position of the assigned zeros and also the RL can be chosen almost freely (it must be smaller than the natural RL relative to the TZ closest to the passband; it should also be not too close to the natural RL otherwise a solution with strongly degraded stopband attenuation may be found).

The choice of the number $n c$ of complex RZs depends on the assigned TZs: a complex RZ allows for two additional degrees of freedom, that can be exploited for the extraction of up to two $\mathrm{TZs}$ from either one of the dangling branches. In case a single $\mathrm{TZ}$ is extracted from a dangling branch, the added RZ can be real (one degree of freedom is enough). When both dangling branches extract one TZ each, two added real RZs are needed. Real reflection zeros can be used also for 3 or $4 \mathrm{TZs}$ if specific symmetries exist in the TZs pattern.

Also in this case, the evaluation of the polynomials determining the new characteristic function requires the solutions of a non-linear system expressing the constraint (6), with the unknows represented by the real and imaginary part of the $n c$ transmission zeros. Being the solution method optimization-based, the initial assignment of the unknowns may affect both the convergence of the solution and the final result obtained. In any case, we always had convergence in all the performed tests, although we observed that the initial unknowns assignment affects the overall computation time to some extent (the computation time has however never exceeded a few seconds on a $\mathrm{PC}$ with average computing power).

As a first synthesis example using the new characteristic, the first case used above with the Bounded Chebyshev characteristic has been considered. With two asymmetric zeros extracted from the source, a single complex zero is required $(n c=1)$, whose value is assigned initially to $z C=1+\mathrm{j} 0$ (the two unknowns of the non-linear problem are initialized to $[1,0])$.
The imposed return loss is $R L=18 \mathrm{~dB}$ and the assigned zeros are $[-1.1 \mathrm{i}, 1.2 \mathrm{i}]$ (the natural $R L$ relative to the zero at $-1.1 \mathrm{j}$ is 20.3 $\mathrm{dB})$. The solution found for the complex zero is ${ }_{z} C_{\text {opt }}=0.2545+0.657 \mathrm{i}$ that determines the Reduced Chebycheff response with the following imaginary reflection zeros: [0.9791i, $0.73 \mathrm{i},-0.986 \mathrm{i},-0.846 \mathrm{i},-0.485 \mathrm{i}, 0.0623 \mathrm{i}]$. The response is shown in Fig. 9a, compared with the one obtained with the GC characteristic of order 7. As we can see, the attenuation with the Reduced Chebyshev characteristic is somewhat smaller, especially in the upper stopband (but slightly better than the one with the Bounded Chebycheff characteristic with no NRN).

The following example considers the second case used for the Bounded Chebyshev characteristic $\left(n p=8, \Omega_{z}^{S}=[-1.25,1.1]\right.$, $\left.\Omega_{z}^{L}=[-1.45,1.225]\right)$. Note that in this case a solution is possible without the use of NRNs. Moreover, we can assign the return loss below the natural RL of the closest $\mathrm{TZ}$ to the passband (20.3 dB), improving consequently the attenuation in the stopbands. We have then assigned $\mathrm{RL}=18 \mathrm{~dB}$.

Being four transmission zeros assigned, we need two complex zeros that are initialized to $z C=[1+0 \mathrm{i},-1+0 \mathrm{i}]$. The solution of the non-linear problem gives the optimized complex zeros: $z C_{\text {opt }}=[0.3155-0.7327 \mathrm{i},-1.0032-0.4646 \mathrm{i}]$. The imaginary reflection zeros produced by the Reduced Chebyshev characteristic with these complex zeros result: $z F=[-0.9794 i$, -0.7245i, 0.99061i, 0.8959i, 0.6086i, 0.0307i]. The overall computation time (including the evaluation of the characteristic polynomials) was a few seconds on a PC with Intel core I7 processor

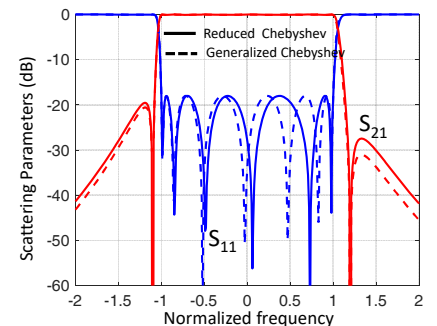

(a)

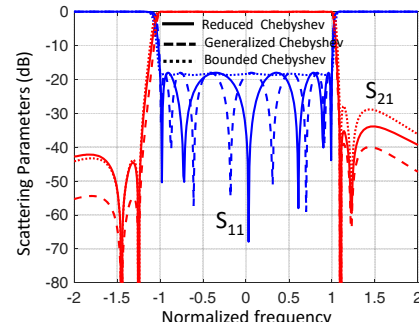

(b)
Fig. 9. (a) Filter with $n p=7, \Omega_{z S}=[-1.1,1.2]$. Solid lines: Reduced Chebyshev characteristic, one added RZ. Dashed lines: Chebyshev characteristic of order 7. (b) Filter with $n p=8, \Omega_{z s}=[-1.25,1.1], \Omega_{z L}=[-1.45,1.225]$. Solid lines: Reduced Chebyshev characteristic, two added RZs. Dashed lines: Chebyshev characteristic of order 8. Dotted lines: Bounded Chebyshev characteristic of order 8 and $R L_{\min }=18.3 \mathrm{~dB}, R L_{\max }=18.67 \mathrm{~dB}$.

The S parameters obtained with the computed characteristic polynomials are shown in Fig. 9b (solid lines), compared with the one determined by the GC characteristic of order 8 (dashed lines). In this case, the reduction of attenuation caused by the complex RZs is still more evident. With the Bounded Chebyshev characteristics (and a NRN at source), there is a sequence of signs of the real part of RZs that gives $R L_{\min }=18.3 \mathrm{~dB}, R L_{\max }=18.67 \mathrm{~dB}$. The attenuation produced is however worse than that obtained with the Reduced Chebyshev characteristic (Fig. 9b, dotted lines).

In conclusion, the Reduced Chebyshev characteristic allows maximum flexibility in the assignment of TZs and RL in the synthesis of path filters without NRNs. Compared to the GC 
characteristic (for the same order $n p$ and passband $R L$ ) there is a reduction of the stopband attenuation which is the price to pay for a true inline realization exhibiting a response with arbitrarily placed TZs.

\section{SyntheSis OF PATH FILTERS LOWPASS PROTOTYPE}

Once the characteristic polynomials are known, the synthesis of the low-pass prototype network (defining the normalized coupling matrix of the filter) is in general carried out by means of well-known methods, usually based on suitable matrix manipulations [20]. The most usual approach consists in the evaluation of the coupling matrix of the canonical transversal prototype, followed by a suitable sequence of matrix rotations that produce in the end the coupling matrix of the desired topology. However, in case of path filter, the synthesis of the transversal prototype as proposed in [20] fails because of the presence of matrix residues of rank 2 in the admittance matrix. We have then developed an alternative procedure that allows the direct synthesis of the path topology, without requiring the computation of the transversal coupling matrix.

Suppose that we have in mind the realization of a path filter, with $n^{S}$ distinct TZs $j \Omega_{z, k}^{S}$ to be generated by the source dangling branch, and $n^{L}$ distinct TZs $j \Omega_{z, k}^{L}$ to be generated by the dangling branch at load. Suppose also that we have obtained a lossless $2 \times 2$ scattering response $S$ such that $S_{11}\left(j \Omega_{z, k}^{S}\right)=-1$ and $S_{22}\left(j \Omega_{z, k}^{L}\right)=-1$ (from (4)).

With reference to the compact model of path filter shown in Fig. 2a (Section II), the input admittance $Y_{\text {in }}$ is then given by:

$$
Y_{\text {in }}=\frac{\left(1-S_{11}\right)}{\left(1+S_{11}\right)}=\frac{E-F}{E+F}
$$

It can readily see that $Y_{\text {in }}$ results of the parallel connection of $Y^{S}$ with the input admittance of to the two-port response $S^{D} \odot S^{L}$, that is, by (3):

$$
\begin{aligned}
Y_{\text {in }}=Y^{S} & +\frac{1-\left[S^{D} \odot S^{L}\right]_{1,1}}{1+\left[S^{D} \odot S^{L}\right]_{1,1}}= \\
& =j a^{S}+\sum_{k=1}^{n^{S}} \frac{r_{k}^{S}}{S-j \Omega_{z, k}^{S}}+\frac{1-\left[S^{D} \odot S^{L}\right]_{1,1}}{1+\left[S^{D} \odot S^{L}\right]_{1,1}}
\end{aligned}
$$

The condition $S_{11}\left(j \Omega_{z, k}^{S}\right)=-1$ ensure that $Y_{\text {in }}$ has simple poles at $\left\{\Omega_{z, k}^{S}\right\}$ so that each $r_{k}^{S}$ can be computed as the residue of $Y_{\text {in }}$ at $s=j \Omega_{z, k}^{S}$ and:

$$
j a^{S}=\lim _{s \rightarrow \infty}\left(Y_{i n}(s)\right)=\frac{1-S_{11}(\infty)}{1+S_{11}(\infty)}
$$

Note that in the case $S_{11}(\infty) \neq 1$ we have $a^{S} \neq 0$ and a frequencyinvariant susceptance (that is an NRN of value $j a^{\mathrm{S}}$ ) appears in parallel to source (or load in case $S_{22}$ is considered). This happens when a phase term is introduced in the definition of the characteristic polynomials (as seen Section II).

Once $Y^{S}$ is evaluated, $S^{S}$ can be obtained from (3) and, similarly, also $S^{L}$ can be computed from $Y^{L}$. Eventually $S^{D}$ can be determined by proper left and right extraction of $S^{S}$ and $S^{L}$ from $S$ [13].

The realization of $S^{D}$ as a coupled resonator circuit is classical. The representation of $Y^{S}$ (or $Y^{L}$ ) as a circuital branch (see Fig. 10) follows a similar technique, remarking that (2) is associated to the transversal form of this branch by:

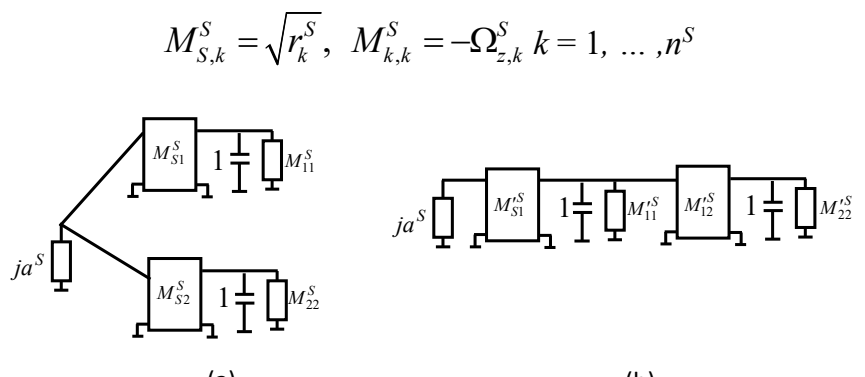

(a)

(b)

Fig. 10. (a) Dangling branch transversal form (b) Dangling branch in inline form

In case of a branch of order 1, transversal and inline forms coincide. For order 2 branches (the maximum allowed in this work), a simple matrix rotation allows to derive the parameters of the inline branch from those of the transversal branch:

$$
\begin{aligned}
& \theta=-\tan ^{-1}\left(M_{S 2}^{S} / M_{S 1}^{S}\right), \quad c_{r}=\cos (\theta), \quad s_{r}=\sin (\theta) \\
& M_{S 1}^{\prime S}=c_{r} M_{S 1}^{S}-s_{r} M_{S 2}^{S}, \quad M_{12}^{\prime S}=s_{r} c_{r}\left(M_{11}^{S}-M_{22}^{S}\right) \\
& M_{11}^{\prime S}=c_{r}^{2} M_{11}^{S}+s_{r}^{2} M_{22}^{S}, \quad M_{22}^{\prime S}=s_{r}^{2} M_{11}^{S}+c_{r}^{2} M_{22}^{S}
\end{aligned}
$$

As an example of this realization procedure, we detail the synthesis of the GC path filter presented in ref. [18] with $n p=10$ and two symmetric TZs located at $\pm j 1.1327$ in the normalized frequency axis. Note that this filter has been actually fabricated in coaxial technology and was used in [18] for validating the Path Filter concept.

The natural RL relative to the assigned pair of TZs, computed through the procedure given in section II, is $23.44 \mathrm{~dB}$. The characteristic polynomials $P(s), F_{0}(s), E_{0}(s)$ that characterize the correspondent equiripple response and define the global scattering matrix $S$ are reported in Table I. The polynomials $m^{S}(s)$ and $q^{S}(s)$ defining the input admittance $Y_{\text {in }}=\left(E_{0}-F_{0}\right) /\left(E_{0}+F_{0}\right)=m^{S}(s) / q^{S}(s)$ are given in Table I.

The frequency response computed in the normalized domain is plotted in Fig. 11.

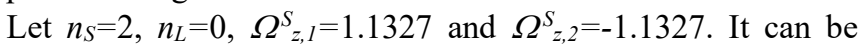
verified that both $j \Omega_{z, 1}^{S_{z}}$ and $j \Omega_{z, 2}^{S_{2}}$ are poles of $Y_{i n}$. The correspondent residues $r_{1}^{S}$ and $r_{2}^{S}$ of $Y_{\text {in }}$ are both equal to $\frac{m^{S}( \pm 1.1327 j)}{q^{S}( \pm 1.1327 j)}=0.2258$ and, after setting $a^{S}=0$ (no NRN at $\mathrm{S}$ node), we can compute the one-port admittance $Y^{S}(S)$ of the source dangling branch using (1) and the corresponding twoport scattering matrix $S^{S}(s)$ with (2).

Finally, the polynomials defining the scattering matrix $S^{D}(s)$ of the mainline filter are obtained from left extraction of $S^{S}$ from $S$, using the technique in [13]. The results of this process are given in Table II. 
TABLE I

ChaRACTERISTIC POLYNOMIALS OF THE DESIGNED FiLTER

\begin{tabular}{|l|l|l|l|l|l|}
\hline degree & $P(s) / j$ & $F_{0}(s)$ & $E_{0}(s)$ & $m^{S}(s)$ & $q^{S}(s)$ \\
\hline 10 & 0 & 1 & 1 & 2 & 0 \\
\hline 9 & 0 & 0 & 2.19016 & 2.19016 & 2.19016 \\
\hline 8 & 0 & 2.68044 & 5.07884 & 7.75928 & 2.3984 \\
\hline 7 & 0 & 0 & 6.86947 & 6.86947 & 6.86947 \\
\hline 6 & 0 & 2.55652 & 8.29684 & 10.85336 & 5.74032 \\
\hline 5 & 0 & 0 & 7.35811 & 7.35811 & 7.35811 \\
\hline 4 & 0 & 1.01901 & 5.44358 & 6.46259 & 4.42457 \\
\hline 3 & 0 & 0 & 3.04268 & 3.04268 & 3.04268 \\
\hline 2 & 0.041803 & 0.147345 & 1.29113 & 1.438475 & 1.143785 \\
\hline 1 & 0 & 0 & 0.365043 & 0.365043 & 0.365043 \\
\hline 0 & 0.053632 & 0.003617 & 0.053753 & 0.05737 & 0.050136 \\
\hline
\end{tabular}

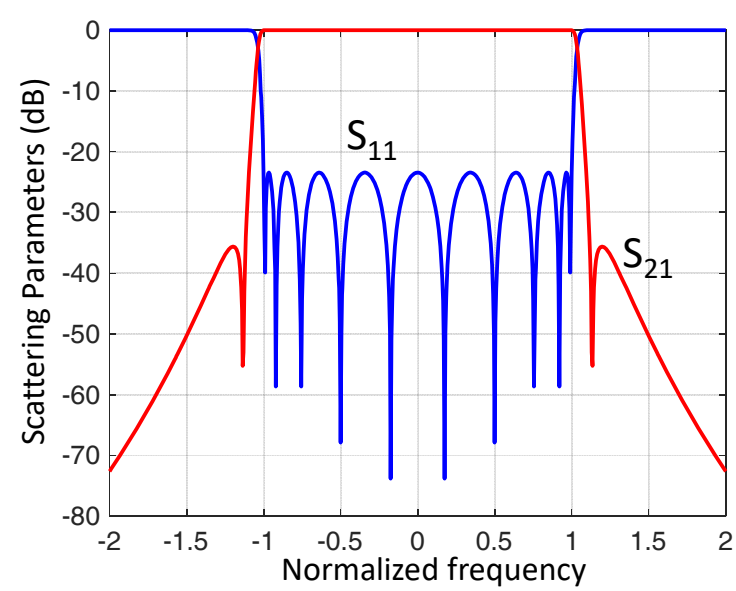

Fig. 11. Filter response in the normalized domain (from [18].)

TABLE II

COEFFICIENTS OF MATRIX $\mathrm{S}^{\mathrm{D}}$ AND $\mathrm{S}^{\mathrm{S}}$ POLYNOMIALS. $\mathrm{F}^{\mathrm{D}}(\mathrm{S})$ REPRESENTS THE NUMERATOR OF $\mathrm{S}^{\mathrm{D}}{ }_{11}, \mathrm{P}^{\mathrm{D}}(\mathrm{S})$ THE NUMERATOR OF $\mathrm{S}^{\mathrm{D}}{ }_{12}$ AND $\mathrm{E}^{\mathrm{D}}(\mathrm{S})$ THE DENOMINATOR OF $\mathrm{S}^{\mathrm{D}}$. SIMILAR NOTATION FOR $\mathrm{S}^{\mathrm{S}}$.

\begin{tabular}{|l|l|l|l|l|l|l|}
\hline deg. & $P^{D}(s) / j$ & $F^{D}(s)$ & $E^{D}(s)$ & $P^{S}(s)$ & $F^{S}(s)$ & $E^{S}(s)$ \\
\hline 8 & 0 & 0.15526 & 0.1553 & 0 & 0 & 0 \\
\hline 7 & 0 & -0.0698 & 0.2696 & 0 & 0 & 0 \\
\hline 6 & 0 & 0.29362 & 0.5120 & 0 & 0 & 0 \\
\hline 5 & 0 & -0.0917 & 0.5372 & 0 & 0 & 0 \\
\hline 4 & 0 & 0.16226 & 0.4873 & 0 & 0 & 0 \\
\hline 3 & 0 & -0.0286 & 0.3043 & 0 & 0 & 0 \\
\hline 2 & 0 & 0.02526 & 0.1430 & 0.609 & 0 & 0.6094 \\
\hline 1 & 0 & -0.0012 & 0.4283 & 0 & -0.137 & 0.1371 \\
\hline 0 & $-6.47 \mathrm{e}-4$ & $4.39 \mathrm{e}-4$ & $6.49 \mathrm{e}-3$ & 0.781 & 0 & 0.781 \\
\hline
\end{tabular}

The dangling branch at the source is developed using (15) and the all-pole network characterized by $S^{D}$ is developed in in-line form using a classic synthesis approach. The computed elements of the coupling matrix $M$ are reported in the following: $M_{i, i}=0, M_{i, i+1}=[0.67198,1.1327,0,0.6036,0.5261,0.5247$, $0.5312,0.5476,0.59576,0.862,1.0465], \mathrm{M}_{1,4}=0.8022$.

\section{Practical Design Examples}

In this section we will show some examples of how the ideal synthesis of path filters seen in the previous sections can be implemented practically. The aim is to highlight the basic steps necessary to start the dimensioning of the physical filters structure. In practice, once the normalized coupling matrix $M$ has been derived from the scattering polynomials, the denormalized coupling parameters of the filter can be computed as follows [21]:

$$
\begin{aligned}
& f_{k}=f_{0}\left(-\frac{B_{n} M_{k, k}}{2}+\sqrt{\left(\frac{B_{n} M_{k, k}}{2}\right)^{2}+1}\right), \quad k_{i, j}=B_{n} M_{i, j} \\
& Q E_{S, i}=\frac{1}{B_{n} M_{S, i}^{2}}, \quad Q E_{L, j}=\frac{1}{B_{n} M_{L, j}^{2}}
\end{aligned}
$$

where $f_{k}$ represent the resonating frequencies of the cavities, $k_{i, j}$ are the coupling coefficients and $\left(Q E_{S, I}, Q E_{L, j}\right)$ are the external $\mathrm{Q}$ of the resonators coupled to source and load respectively.

Using the above parameters, the dimensioning of the path filter in the desired technology can be carried out with the approach usually adopted for classical inline filters. However, special attention must be paid to the choice of the coupling structures involving source and/or load, so that unwanted couplings do not occur between the cavities adjacent to source and load.

The first example concerns a channel filter for satellite communications in $K a$ band. Assuming $n p=6$, passband 19.7$19.94 \mathrm{GHz}$ and two symmetric TZs at $19.67 \mathrm{GHz}$ and 19.968 $\mathrm{GHz}$, we obtain the topology in Fig. 12 (the de-normalized parameters are reported in the caption). Note that no NRNs or complex RZs are used so the return loss cannot be assigned and results $\mathrm{RL}=27.64 \mathrm{~dB}$. Due to the symmetry, all resonators are synchronous.

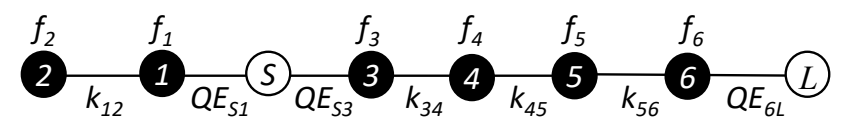

Fig. 12. Routing scheme of the synthesized path filter. Black circles represent the resonators (resonant frequency $f_{k}$ ), solid lines are the couplings (coupling coefficients $k_{i, j}$ or external Q $Q E_{i, j}$ ). (or the external Q in case of the couplings with source or load). The following values are obtained from the synthesis: $f_{k}=19.82 \mathrm{GHz}, k_{12}=0.0149, k_{34}=0.0081, k_{45}=0.0078, k_{56}=0.0116, Q E_{S I}=142.95$, $Q E_{S 3}=115.75, Q E_{6 L}=63.96$.

The filter has been dimensioned in rectangular waveguide with $a=12.95 \mathrm{~mm}, b=8.5 \mathrm{~mm}$. The cavities operate on $\mathrm{TE}_{101}$ mode and the couplings are realized with inductive irises. The parameters of the filter equivalent circuit (Fig. 13a) can be derived by exploiting the definition of the coupling coefficient. Assuming all the series resonators characterized by the same equivalent reactance, the coupling inverters are derived as follows:

$$
\begin{gathered}
K_{i, j}=k_{i, j} X_{e q}, \quad K_{S, 1}=\sqrt{\frac{X_{e q}}{Q_{S, 1}}}, \\
K_{S, 3}=\sqrt{\frac{X_{e q}}{Q_{S, 3}}}, \quad K_{L, 6}=\sqrt{\frac{X_{e q}}{Q_{L, 6}}}
\end{gathered}
$$




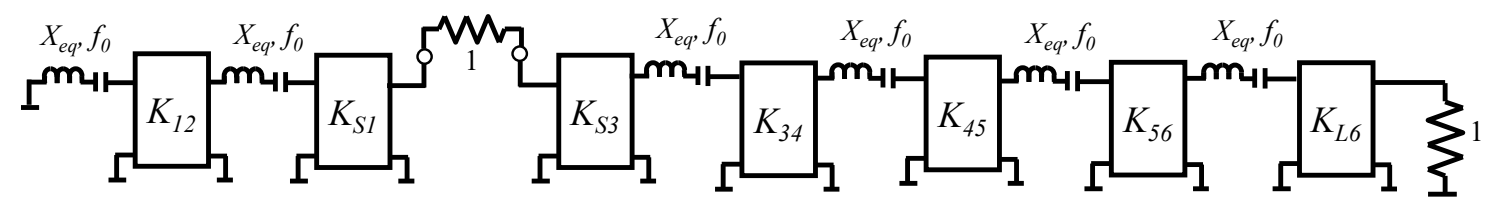

(a)

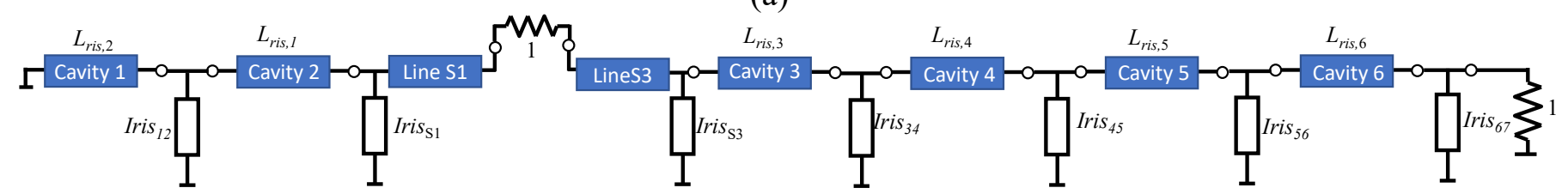

(b)

Fig. 13. (a): De-normalized equivalent circuit of the path filter (the rectangles represent impedance inverters whose value is computed by (14). (b). Scheme of the filter with irises replacing the impedance inverters. Due to the loading effects of the irises, the lengths of the cavities are no longer all equal.

where the equivalent reactance $\left(X_{e q}\right)$ is determined by the resonant mode of the cavities (whose length is $\lambda_{g 0} / 2$ ) [1]:

$$
X_{e q}=\left.\frac{1}{2} f_{0} \frac{\partial X}{\partial f}\right|_{f=f_{0}}=\frac{\pi / 2}{\sqrt{1-\left(\frac{f_{c}}{f_{0}}\right)^{2}}}
$$

In (18) $X(f)$ is the reactance of a short-circuited waveguide section, half wavelength long and $f_{c}$ is the cutoff frequency of the $\mathrm{TE}_{10}$ mode $(11.58 \mathrm{GHz}$ in the present case). Once the impedance inverters in Fig. 13a are replaced with inductive irises and the series resonators with cavities resonating on $\mathrm{TE}_{101}$ mode, the circuit in Fig. 13b is obtained, where the lengths of the cavities have been suitably modified to account for the irises loading effects [1]. The series connection of the source is realized through an E-plane T-junction with the input waveguide on the broad waveguide wall (the two waveguides sections lineS1 and lineS3 are added on the other arms of the Tjunction to allow the connection of irises S1 and S3).

The geometry of the dimensioned filter is shown in Fig. 14 (the computed dimensions are reported in the caption). Note that cavities 1 and 3 are decoupled by the input T-junction.

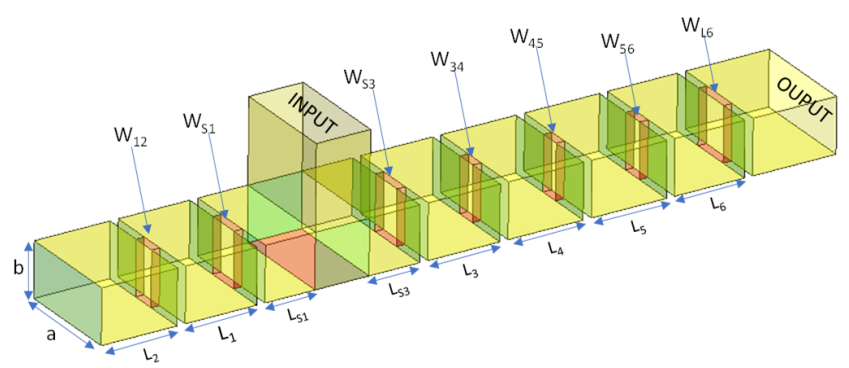

Fig. 14. Geometry of the waveguide path filter. Waveguide cross-section (mm): $\mathrm{a}=12.95, \mathrm{~b}=6.5$. $\mathrm{L}_{\mathrm{i}}$ represent lengths of waveguide sections in $\mathrm{mm} ; \mathrm{W}_{\mathrm{i}, \mathrm{j}}$ are the irises opening in $\mathrm{mm}$. Irises thickness is $1 \mathrm{~mm}$. The dimensioning gives the following results: $\mathrm{L}_{1}=8.624, \mathrm{~L}_{2}=9.069, \mathrm{~L}_{\mathrm{S} 1}=6.072, \mathrm{~L}_{\mathrm{S} 3}=6.004, \mathrm{~L}_{3}=8.636$, $\mathrm{L}_{4}=8.986, \quad \mathrm{~L}_{5}=8.943, \quad \mathrm{~L}_{6}=8.266 ; \quad \mathrm{W}_{12}=2.975, \quad \mathrm{~W}_{\mathrm{Sl}}=3.841, \quad \mathrm{~W}_{\mathrm{S} 3}=4.082$, $\mathrm{W}_{34}=2.497, \mathrm{~W}_{45}=2.468, \mathrm{~W}_{56}=2.775, \mathrm{~W}_{\mathrm{L} 6}=5.002$.

The filter response, computed by full wave analysis (Mician Microwave Wizard ${ }^{\circledR}$ ), is show in Fig. 15.

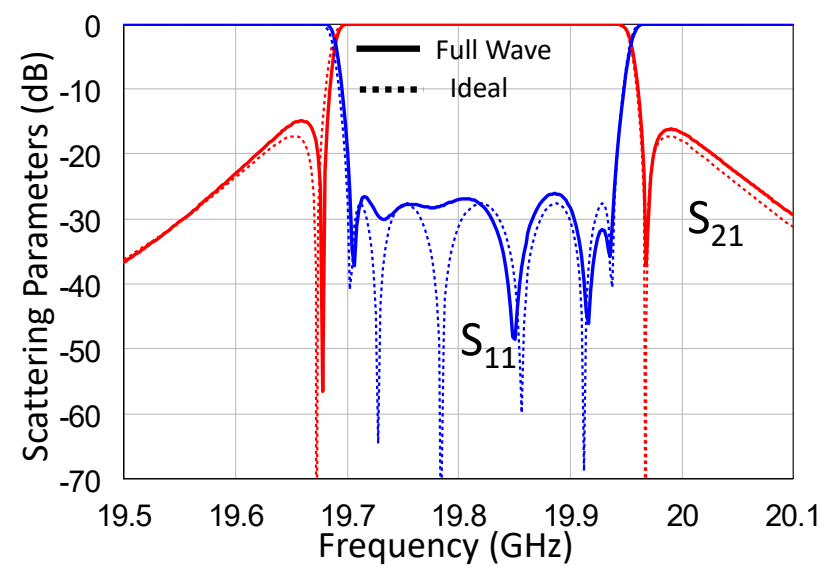

Fig. 15. Computed waveguide filter response (full wave analysis). The ideal polynomial response of the synthesized prototype is reported for comparison.

Note that no full wave optimization of the response has been performed (the dimensions are those obtained by the initial dimensioning based on the filter equivalent circuit).

The return loss of path filters with symmetric response such as the one just considered cannot be freely assigned. High values of RL however penalize the attenuation in the filter stopbands, so it may be convenient to reduce RL (if the specific application tolerates it). As we have seen in Section III, this can be achieved by introducing a real reflection zero. In general, when a complex reflection zero replaces an imaginary one in the filter characteristic, the stopband attenuation decreases (for the same return loss). But if $R L$ is noticeably reduced, an increase of the filter rejection is obtained as well. To illustrate this possibility, let suppose that the filter we have designed just tolerates $20 \mathrm{~dB}$ return loss. We have then re-designed the filter imposing the Reduced Chebyshev characteristic with one real reflection zeros and $R L=20 \mathrm{~dB}$. The new values of the parameters of the synthesized filter (same routing scheme of Fig. 12) result in this case: $f_{k}=19.8196 \mathrm{GHz}, k_{12}=0.0149, k_{34}=0.0076, k_{45}=0.0073$, $k_{56}=0.01, Q E_{S 1}=110.3, Q E_{S 3}=110.3, Q E_{6 L}=82.99$.

The dimensioned path filter exhibits the full-wave response shown in Fig. 16 (the new dimensions are reported in the figure caption. Also in this case, no optimization has been performed). Comparing the new response in Fig. 16 with the previous one (Fig. 15), we can observe that, despite the introduction of a real 
$\mathrm{RZ}$, the attenuation in the stopbands has increased by about 4 $\mathrm{dB}$ (with $R L$ reduced from 27.6 to $20 \mathrm{~dB}$ ).

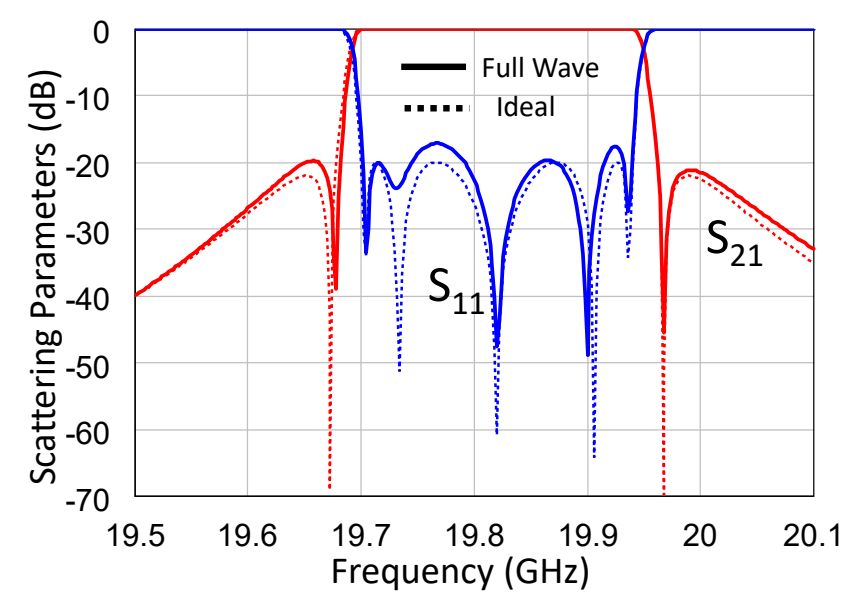

Fig. 16. Full wave response of the re-dimensioned waveguide filter (solid lines). The polynomial response (Reduced Chebyshev characteristic) is reported for comparison (dashed lines). The computed dimensions $(\mathrm{mm})$ are reported in the following. Lris 1 -Lris 6 : [8.561, 9.071, 8.627, 8.998, 8.969, 8.366], $\mathrm{L}_{01}$ : 5.98, $\mathrm{L}_{03}=6.045, \mathrm{~W}_{01}: 4.053, \mathrm{~W}_{01}: 4.129, \quad \mathrm{~W}_{12}=2.976, \quad \mathrm{~W}_{34}=2.46, \mathrm{~W}_{45}=2.42$, $\mathrm{W}_{56}=2.661, \mathrm{~W}_{6 \mathrm{~L}}=4.801$

Another example of waveguide path filter has been designed with $n p=7$ and 3 transmission zeros, two extracted by the dangling branch on the source $(19.67,19.968 \mathrm{GHZ})$ and one by the dangling branch on the load $(19.997 \mathrm{GHz})$. The synthesis of the prototype has been performed by using also in this case the Reduced Chebyshev characteristic with two complex reflection zeros and $R L=20 \mathrm{~dB}$.

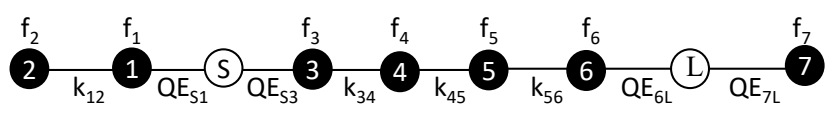

Fig. 17. Routing scheme of the waveguide path filter with $n p=7$ and $3 \mathrm{TZs}$. Synthesis result (frequencies in $\mathrm{GHz}$ ): $f_{1}=19.826, f_{2}=19.813, f_{3}=19.816$, $f_{4}=19.814, \quad f_{5}=19.802, \quad f_{6}=19.741, \quad f_{7}=19.997 ; \quad k_{12}=0.0149, \quad k_{34}=0.0075$, $k_{45}=0.0071, k_{56}=0.0097, Q E_{S I}=107.0, Q E_{S 3}=111.89, Q E_{6 L}=60.96, Q E_{7 L}=107.37$.

The routing scheme of the de-normalized prototype obtained from the synthesis is shown in Fig. 17 and the dimensioned waveguide structure is reported in Fig. 18. The computed response by full wave analysis (Mician Microwave Wizard ${ }^{\circledR}$ ) is shown in Fig.19 (also the ideal polynomial response is reported for comparison). The expected filter performances are confirmed by the simulations also in this case (again, no optimization has been performed after the initial dimensioning).

In conclusion, we can observe that the implementation of path filters in waveguide technology is relatively easy and convenient. In fact, the same dimensioning procedure employed for classical inline filters with inductive irises can be adopted. Moreover, the TZs can be placed very close to the passband and their position is controlled with high accuracy.

We conclude this Section by observing that also other technologies can be advantageously employed for the fabrication of path filters. For instance, we have designed and verified successfully (by full wave simulations) a test filter in microstrip technology. In [18] a path filter in coaxial technology has been designed and fabricated; it was used as an experimental validation the path filter concept. As the present work is an expanded version of [18] we have not repeated here this validation.

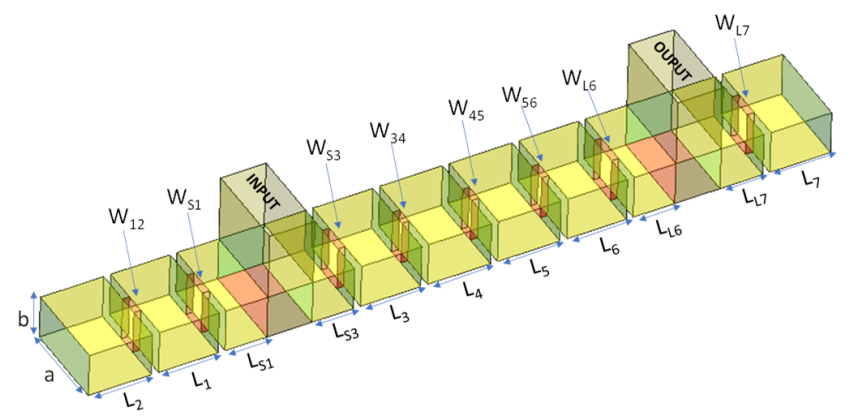

Fig. 18. Dimensioned waveguide path filter with $n p=7$ and 3 transmission zeros (all dimensions in $\mathrm{mm}$ ). Waveguide cross-section $(\mathrm{mm})$ : $\mathrm{a}=12.95, \mathrm{~b}=6.5$. The irises thickness is $1 \mathrm{~mm}$. Computed values $(\mathrm{mm}): \mathrm{L}_{1}=8.555, \mathrm{~L}_{2}=9.075$, $\mathrm{L}_{\mathrm{S} 1}=5.982, \mathrm{~L}_{\mathrm{S} 3}=5.976, \mathrm{~L}_{3}=8.643, \mathrm{~L}_{4}=9.006, \mathrm{~L}_{5}=8.986, \mathrm{~L}_{6}=8.524, \mathrm{~L}_{\mathrm{L} 6}=5.972$, $\mathrm{L}_{\mathrm{L} 7}=6.149, \quad \mathrm{~L}_{7}=8.702 ; \quad \mathrm{W}_{12}=2.978, \quad \mathrm{~W}_{\mathrm{S} 1}=4.059, \quad \mathrm{~W}_{\mathrm{S} 3}=4.101, \quad \mathrm{~W}_{34}=2.439$, $\mathrm{W}_{45}=2.412, \mathrm{~W}_{56}=2.264, \mathrm{~W}_{\mathrm{L} 6}=4.536, \mathrm{~W}_{\mathrm{L} 7}=3.987$.

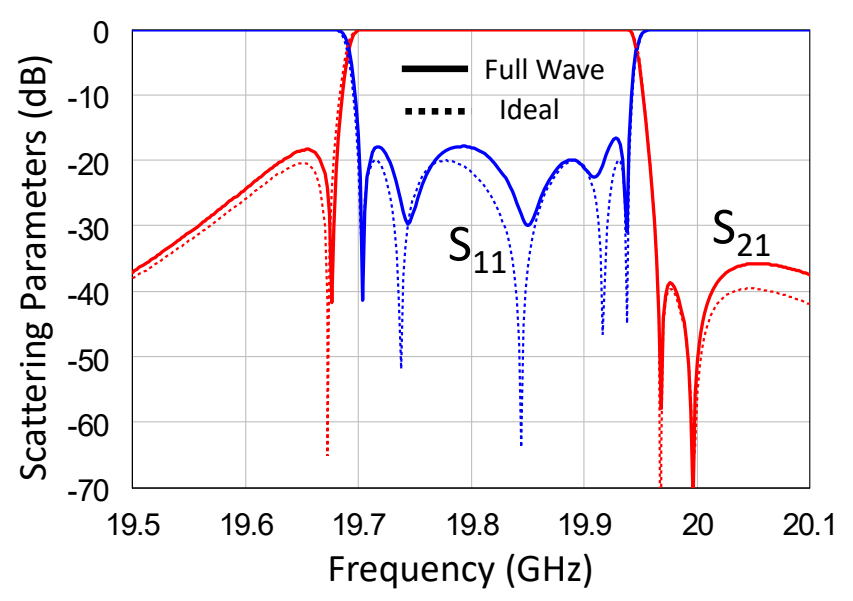

Fig. 19. Full wave response of waveguide filter with $\mathrm{np}=7$ and $3 \mathrm{TZs}$ (solid lines). The ideal polynomial response (Reduced Chebyshev characteristic) is reported for comparison (dashed lines).

\section{CONCLUSIONS}

In this paper, we have presented a general approach to the synthesis and design of path filters. First the general conditions that the polynomial model of the path filter must satisfy have been derived. These conditions require one or more additional constraints on the equiripple characteristic function defining the filter response. With the GC characteristic only a degree of freedom is available (represented by the RL in the passband), then only one transmission zero can be arbitrarily assigned (also two or four symmetric and/or coincident zeros are possible). The return loss level cannot however be freely assigned. To increase the design flexibility, NRNs can be added to source and/or load. In this way two or three asymmetric zeros can be assigned but the return loss is still not freely assignable. A method has then been shown to further increase the design flexibility, which consists in removing the condition of purely imaginary reflection zeros. Two new characteristic functions 
(namely the Bounded Chebyshev and the Reduced Chebyshev characteristics) have consequently been introduced, featuring complex reflection zeros. The first is still limited in the arbitrary assignment of TZs and RL (use of NRNs increases the flexibility but increases as well the implementation complexity of the filter structure). The second allows the maximum flexibility in the assignment of the TZs and RL, and the use of NRNs is not required. However, the return loss must not exceed a threshold determined by the assigned TZs and the stopband attenuation is reduced with respect the GC characteristic. In conclusion, the most convenient choice among the possible three is the $\mathrm{GC}$ when the $\mathrm{TZ}$ can be assigned with the required symmetry and the resulting return loss is not too large. If this is not possible, the best choice is the Reduced Chebyshev, especially when NRNs are not allowed. If a RL larger than the minimum allowed by the Reduced Chebyshev is required (this happens with TZs very close to the passband), the Bounded Chebyshev characteristic can be used, possibly including NRNs when the assigned TZs do not respect the required symmetry constraints.

An ad hoc procedure for the synthesis of path filter has also been given, allowing to overcome the limitations that more general synthesis techniques might suffer from when applied to path filters. Finally, several simulated examples of path filters in waveguide technology have demonstrated how the dimensioning of this filters class is very similar to the one used for all-pole filters.

\section{APPENDIX A}

\section{BOUNDED CHEBYSHEV CHARACTERISTIC FUNCTIONS}

The approximation function in the normalized frequency domain $s=j \Omega$ can be expressed as follows:

$$
A(\Omega)=1+\varepsilon_{\min }^{2} C_{n}^{2}(\Omega)=\frac{1}{\left|S_{21}\right|^{2}}
$$

where $\varepsilon_{\text {min }}$ is related to the minimum return loss $R L_{\text {min }}$ in the passband: $\varepsilon_{\min }^{2}=\left(10^{R L \min / 10}-1\right)^{-1}$. The usual form of the characteristic function $C_{n}(\Omega)$ is the so called Generalized Chebyshev characteristic whose mathematical definition can be found in [20]. $C_{n}(\Omega)$ oscillates between -1 and 1 in the passband $(-1<\Omega<1)$, so that $A(\Omega)$ is equal 1 at the frequencies $\Omega_{r, i}$ where $\mathrm{C}_{n}\left(\Omega_{r, i}\right)=0$. At these frequencies, $S_{11}\left(\Omega_{r, i}\right)=0$ and $C_{n}^{2}(\Omega)$ has minima equal to zero : $C_{n}^{2}\left(\Omega_{r, i}\right)=C_{\min }^{2}=0$.

Here we introduce a new characteristic function $C_{n}^{\prime}(\Omega)$ (namely the Bounded Chebyshev characteristic) whose magnitude is assumed to be greater than 0 at $\Omega_{r, i}$, so that $C_{n}^{\prime 2}(\Omega)$ oscillates between 1 and $C_{\min }^{\prime 2}$ for $-1<\Omega<1$. We get $C_{n}^{\prime 2}(\Omega)$ by suitably scaling $C_{n}^{2}(\Omega): C_{n}^{\prime 2}(\Omega)=\frac{\alpha+C_{n}^{2}(\Omega)}{1+\alpha}$, with $C_{\min }^{\prime 2}=\frac{\alpha}{1+\alpha}$. Note that $C_{n}^{\prime 2}( \pm 1)=1$, as required.

The new characteristic function can be expressed as:

$$
\varepsilon_{\min }^{2} C_{n}^{\prime 2}(\Omega)=\frac{\left|S_{11}\right|^{2}}{\left|S_{21}\right|^{2}}=\frac{|F(j \Omega)|^{2}}{|P(j \Omega)|^{2}}=\varepsilon_{\min }^{2} \frac{\alpha+C_{n}^{2}(\Omega)}{1+\alpha}
$$

Imposing the value $C_{\min }^{\prime 2}=\alpha /(1+\alpha)$ at the minima frequencies $\left\{\Omega_{r, i}\right\}$ of the Chebyshev characteristic we find:

$$
\alpha=\frac{\varepsilon_{\max }^{2}}{\varepsilon_{\min }^{2}-\varepsilon_{\max }^{2}}
$$

where $\varepsilon_{\max }^{2}=\left(10^{R L \max / 10}-1\right)^{-1}$ and $R L_{\max }$ is the maximum value of the return loss obtained with the new characteristic function $\left(R L_{\min }\right.$ is the value of $R L$ defining $\left.\varepsilon_{\min }\right)$.

Note that we can replace $\varepsilon_{\min }^{2} C_{n}^{2}(\Omega)=\left|F_{0}(j \Omega) / P_{0}(j \Omega)\right|^{2}$ in (A2), with $F_{0}$ and $E_{0}$ the polynomials defining the Chebyshev characteristic (A1). Then, applying analytic continuation, we obtain:

$$
k \cdot F(s) F(s)^{*}=\varepsilon_{\min }^{2} \alpha P_{0}(s) P_{0}(s)^{*}+F_{0}(s) F_{0}(s)^{*}
$$

where $k$ is a real constant. The roots of the right-hand side of (A4) are in complex para-conjugate pairs. Selecting one root per pair with an arbitrary sign of the real part, we obtain the roots of $F(s)$, which is finally defined, being a monic polynomial.

The transmission polynomial $P(s)$ can be expressed as $P(s)=p_{0} P_{0}(s)$, with the monic polynomial $P_{0}(s)$ defined by the transmission zeros. The constant $p_{0}$ can be determined by imposing that $C_{n}^{\prime 2}=1$ for $s= \pm j$. From (A2) we get:

$$
p_{o}^{2}=\frac{|F( \pm j)|^{2}}{\varepsilon_{\min }^{2} C_{n}^{\prime 2}( \pm 1)\left|P_{n}( \pm j)\right|^{2}}=\frac{|F( \pm j)|^{2}}{\varepsilon_{\min }^{2}( \pm j)\left|P_{n}( \pm j)\right|^{2}}
$$

Finally, the polynomial $E(s)$ is obtained from $P(s)$ and $F(s)$ through the Feldtkeller equation and spectral factorization [20]. We observe that, for assigned $R L_{\min }$ and $R L_{\max }$, there are $2^{n p}$ possible ways to select the roots of $F(s)$ from those of $F(s) F(s)^{*}$ (depending on the choice of the sign of their real part). All the resulting $F(s)$ produce the same $\left|\mathrm{S}_{11}\right|$ and $\left|\mathrm{S}_{22}\right|$ (lossless condition imposes $\left.\left|S_{11}\right|=\left|S_{22}\right|\right)$. The phases of $S_{11}$ and $S_{22}$ are however different because the roots of $F(s)$ are not imaginary so that $F(s) \neq F(s)^{*}$. We can exploit this choice and the parameter $R L_{\max }$ as an additional degree of freedom in the synthesis of path filters.

As an example, consider the following assignment: $n p=8$; $\mathrm{TZ}_{\mathrm{s}}=[-1.1 \mathrm{i}$ 1.3i]; $R$ Lmin $=20 \mathrm{~dB}$. Imposing $R$ Lmax $=[25,35,45]$ $\mathrm{dB}$ we get the scattering parameters shown in Fig. A1(a). It can be observed that the position of maxima and minima of $S_{11}$ in the passband are the same of the GC characteristic (to which the new characteristic function tends for $R \operatorname{Lmax} \rightarrow \infty)$. The attenuation in the stopband is smaller with respect the Chebyshev response but the difference is relatively small. 


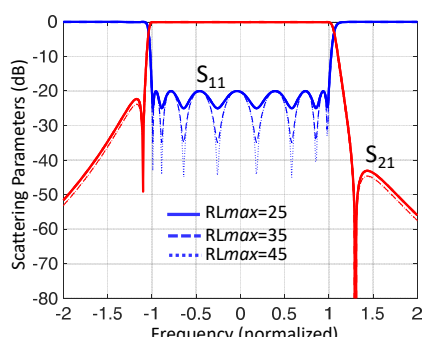

(a) (b)

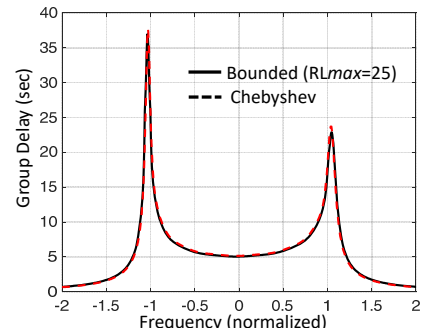

Fig. A1. (a) Scattering parameters of Bounded Chebyshev characteristic for $\mathrm{np}=8, \mathrm{TZs}=[-1.1 \mathrm{i}, 1.3 \mathrm{i}]$ and $\mathrm{RL}=[25,35,45] \mathrm{dB}$. (b): Group Delay of Bounded Chebyshev characteristic for $R L \max =25 \mathrm{~dB}$ ). The response obtained with the $\mathrm{GC}$ Characteristic is reported for reference.

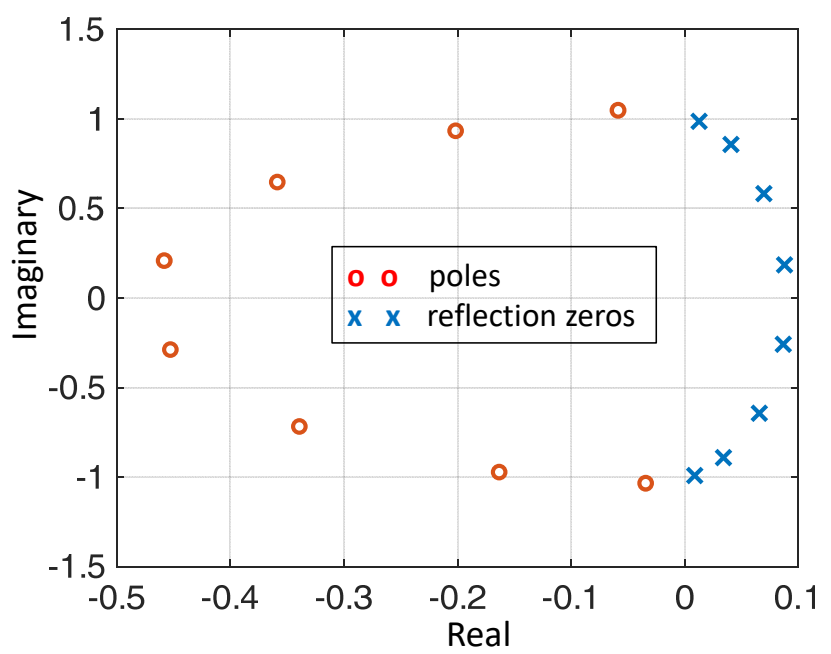

Fig. A2. Representation of the reflection zeros and poles in the complex plane for RL max $=25 \mathrm{~dB}$. Note that the magnitude of $\mathrm{S}_{11}$ is not affected by the sign of the real part of reflection zeros.

In Fig. A1(b) we have reported the computed group delay produced by the Bounded Chebyshev characteristic with $R L \max =25 \mathrm{~dB}$, compared with the GC characteristic. As it can be observed, there are no visible differences between the two curves.

To give an idea of the pole-zero distribution, Fig. A2 shows the pattern of $F(s)$ and $E(s)$ roots for the case $R L_{\max }=25 \mathrm{~dB}$.

\section{APPENDIX B}

\section{REDUCED CHEBYSHEV CHARACTERISTIC FUNCTION}

Let $n p$ be the order of the filter, $n c$ the desired number of additional complex RZs $\left\{z C_{k}\right\}$ (here assigned) and $\left\{j \Omega_{z, k}\right\}$ the set of the $n z$ prescribed TZs (all imaginary). Let $F_{C}(j \Omega)$ the monic polynomial of degree $n c$ having the assigned complex RZs as roots. We then introduce the monic polynomials $P_{0}(\Omega)$ and $F_{0}(\Omega)$ with real roots $\left\{\Omega_{z, k}\right\}$ and $\left\{\Omega_{r, k}\right\}$ respectively. The roots of $P_{0}$ are defined by the $n z$ assigned transmission zeros while the order of polynomial $F_{0}$ is imposed equal to $n p-n c$. We seek the $n p-n c$ unknown coefficients $\left\{f_{k}\right\}$ of $F_{0}(\Omega)$ that make the (real) characteristic function $C(\Omega)=\left|F_{c}(j \Omega)\right| \cdot F_{0}(\Omega) / P_{0}(\Omega)$ equiripple in the passband $(-1 \leq \Omega \leq 1)$.
At this aim, a Remez-like equiripple algorithm, briefly discussed in this appendix, can be used.

To initialize the iteration, let $\left(\Omega_{1}, \ldots, \Omega_{n p-n c-1}\right)$ be the locations of the $n p-n c-1$ in-band maxima of $\left|\mathrm{S}_{11}\right|$ produced by the GC characteristic of order $n p-n c$ with $\left\{j \Omega_{z, k}\right\}$ as TZs. Then proceed as follows:

Step 1) Solve the linear system of $n p-n c+1$ equation in the $n p-n c+1$ unknowns $\left[\left\{f_{k}\right\}, h\right]$ with

$$
\left\{\begin{array}{l}
C(-1)=h \\
C\left(\Omega_{k}\right)=(-1)^{k} h \quad k=1, \ldots, n p-n c-1 \\
C(1)=(-1)^{n p-n c} h
\end{array}\right.
$$

where $h$ is a real number defining the amplitude of the in-band ripple of $C(\Omega)$.

Step 2) With the coefficients $\left\{f_{k}\right\}$ of $F_{0}(\Omega)$ found at the previous step, update the frequencies $\left(\Omega_{1}, \ldots, \Omega_{n p-n c-1}\right)$ of the $n p-n c-1$ extrema of the real function $C(\Omega)$ in the range $-1 \leq \Omega \leq 1$.

Step 3) Using the updated frequencies found in step 2, repeat steps 1 and iterate until the computed frequencies $\left(\Omega_{1}, \ldots, \Omega_{n p-n c-1}\right)$ convergence.

Step 4) The polynomials $P(s), F(s), E(s)$ of the new characteristic (named Reduced Chebyshev) are computed as follows. $P(s)$ is obtained by the assigned TZs $\left\{j \Omega_{z, k}\right\}$ and the scaling constant $p_{0}$ determining the desired RL (see (7)). $F(s)$ is defined by its roots, constituted by the roots of $F_{0}$ (obtained at the end of the procedure) multiplied by $j$ (i.e. $\left\{j \Omega_{r, k}\right\}$ ) and by the assigned complex roots $\left\{z C_{k}\right\}$. Finally, $E(s)$ is obtained from $P(s)$ and $F(s)$ through the Feldtkeller equation and spectral factorization [20].

As an example of application of the described procedure, let consider the second example in Section IV where we assumed: $n p=8, n c=2$, transmission zeros $\left\{j \Omega_{z, k}\right\}=[-1.25 \mathrm{i}, 1.1 \mathrm{i},-1.45 \mathrm{i}$, $1.225 \mathrm{i}], R L=18 \mathrm{~dB}$. The assigned complex reflection zeros are $z C=[-1-0.465 \mathrm{i}, 0.315-0.733 \mathrm{i}]$, that determine the polynomial

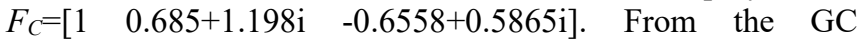
characteristic of order $n p-n c=6$ we get the initial roots of $F_{0}$ $\left\{\Omega_{r, k}\right\}=[-0.9796,-0.7815,-0.263,0.4352,0.8597,0.9883]$. Applying the procedure described above, the following values of the unknowns are obtained: $\left\{f_{k}\right\}=\left[\begin{array}{llll}1 & -0.8229 & -1.4811 & 1.2053\end{array}\right.$ $0.4876-0.39960 .012], h=0.0318$. Fig. B1 shows the characteristic function vs. $\Omega$ at the beginning and at the end of the procedure.

The polynomials of the Reduced Chebycheff characteristic are finally determined as above explained and their coefficients result: $P(s)=[0.25 \mathrm{i},-0.0938,0.78 \mathrm{i},-0.144,0.611 \mathrm{i}] ; F(s)=[1$, $0.685+0.375 \mathrm{i}, 1.811+0.0228 \mathrm{i}, \quad 1.497+1.1087 \mathrm{i}, \quad 0.96+0.0431 \mathrm{i}$, $1.041+0.975 \mathrm{i}, 0.147+0.0123 \mathrm{i}, 0.2262+0.2478 \mathrm{i}, 0.0078-0.007 \mathrm{i}]$ $E(s)=[1, \quad 3.106+0.375 \mathrm{i}, \quad 6.4+0.452 \mathrm{i}, \quad 9.491+0.776 \mathrm{i}$, $10.346+0.4843 \mathrm{i}, 8.826+0.228 \mathrm{i}, 5.4775-0.068 \mathrm{i}, 2.413-0.1285 \mathrm{i}$, $0.6071-0.0703 \mathrm{i}]$. 


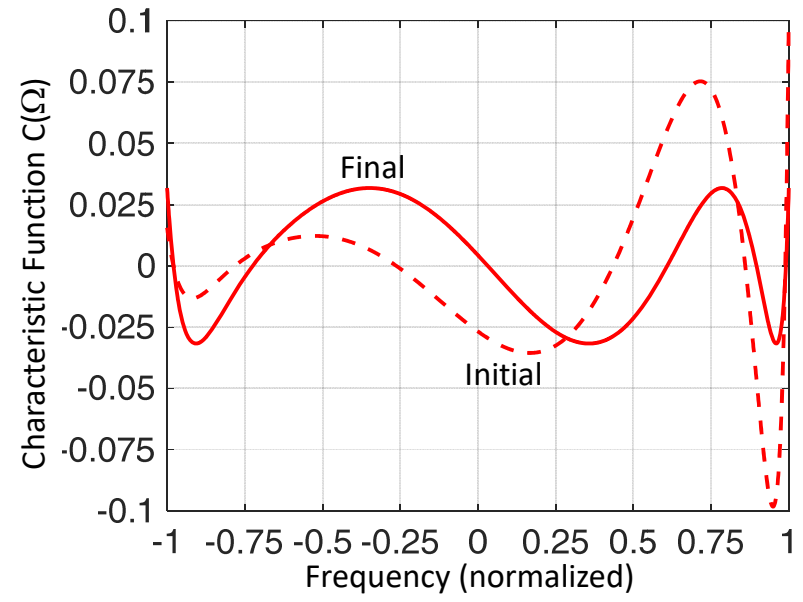

Fig. B1. Characteristic function $\mathrm{C}(\Omega)$ vs. $\Omega$ at the beginning (dashed line) and the end (solid line) of the Remez-like procedure.

The response obtained from $P(s), F(s), E(s)$ (already reported in Fig. 9b, solid line, and therefore not repeated here) confirms the equiripple behavior in the passband. In Fig. B2 the group delay response, compared with the one obtained by the GC characteristic of the same order $(n p)$, is reported.

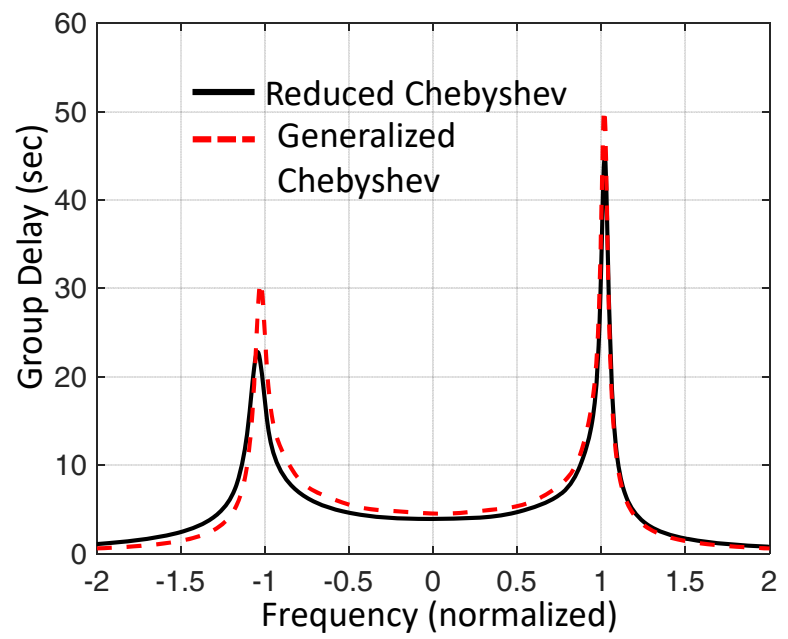

Fig. B2. Group Delay response for the considered example. The result obtained with the $\mathrm{GC}$ characteristic of the same order $(n p=8)$ is reported for reference.

It can be noticed that the Reduced Chebyshev characteristic allows a reduction of the group delay variation in the passband.

The procedure above described provides equiripple passband filter responses of degree $n p$ with $n c$ assigned RZs (provided their real part is not too small) and can be used inside an optimization cycle where the locations of the $n c$ RZs are optimized to meet the pertinent path realizability conditions.

\section{REFERENCES}

[1] G. L. Matthaei, L. Young, and E. M. T. Jones, Microwave FilterImpedance Matching Networks and Coupling Structures. New York: McGraw-Hill, 1964
[2] J. D. Rhodes and R. J. Cameron, General extracted pole synthesis technique with applications to low-loss TE011 mode filters, IEEE Trans. Microw. Theory Tech., vol. 28, n. 9, pp. 1018-1028, Sep. 1980.

[3] J.R. Montejo-Garai., "Synthesis of N-Order Filters with N Transmission Zeros at Real Frequencies by means of Extracted Poles", Electr. Lett., vol.39, n.2, pp. 182-183, Jan. 2003.

[4] S. Amari, G. Macchiarella, "Synthesis of In-Line Filters With Arbitrarily Placed Attenuation Poles by Using Non-Resonating Nodes" IEEE Trans. Microw. Theory Tech., vol. 52, n.10, pp. 3075-3081, Oct. 2005.

[5] Y. Yang, Y. Zeng, M. Yu and Q. Wu, "Synthesis of a New Class of Extracted Pole Filters Without the Ideal Phase Shifters," in IEEE Trans. Microw. Theory Tech., vol. 69, no. 1, pp. 639-646, Jan. 2021.

[6] G. Macchiarella and S. Tamiazzo, "An Application-Oriented Design Procedure for Cascaded-Block Extracted-Pole Filters", in IEEE Trans. Microw. Theory Tech., vol. 69, no. 1, pp. 671-681, Jan. 2021.

[7] S. Amari and J. Bornemann, "Using frequency-dependent coupling to generate finite attenuation poles in direct-coupled resonator bandpass filters", in IEEE Microw. Guided Wave Lett., vol. 9, no. 10, pp. 404-406, Oct. 1999.

[8] M. Politi and A. Fossati, "Direct coupled waveguide filters with generalized Chebyshev response by resonating coupling structures," Proc. 40th Eur. Microw. Conf. (EuMC), pp. 966-969, Paris, Sep. 2010.

[9] L. Szydlowski, N. Leszczynska and M. Mrozowski, "Generalized Chebyshev Bandpass Filters With Frequency-Dependent Couplings Based on Stubs," IEEE Trans. Microw. Theory Techn., vol. 61, no. 10, pp. 3601-3612, Oct. 2013

[10] S. Tamiazzo and G. Macchiarella, "Synthesis of cross-coupled filters with frequency-dependent couplings," IEEE Trans. Microw. Theory Techn., vol. 65, no. 3, pp. 775-782, Mar. 2017.

[11] Y. He, G. Macchiarella, G. Wang, L. Sun, L. Wang and R. Zhang, "A Direct Matrix Synthesis for In-Line Filters with Transmission Zeros Generated by Frequency-Variant Couplings" IEEE Trans. Microw. Theory Techn., vol. 66, N. 4, pp. 1780-1789, Apr. 2018.

[12] Y. He, G. Macchiarella, Z. Ma, L. Sun and N. Yoshikawa, "Advanced direct synthesis approach for high selectivity in-line topology filters comprising N-1 adjacent frequency-variant couplings" IEEE Access, vol. 7, pp. 41659-41668, Mar. 2019.

[13] Y. Zhang, F. Seyfert, S. Amari, M. Olivi and K. -L. Wu, "General Synthesis Method for Dispersively Coupled Resonator Filters With Cascaded Topologies," in IEEE Trans. Microw. Theory Techn., vol. 69, no. 2, pp. 1378-1393, Feb. 2021.

[14] G. Macchiarella, S. Bastioli, R. V. Snyder, "Design of In-line Filters with Transmission Zeros Using Strongly-Coupled Resonators Pairs" IEEE Trans. Microw. Theory Techn., vol. 66 no. 8, pp. 3836-3846, Aug. 2018.

[15] S. Bastioli, R. Snyder, G. Macchiarella, "Design of In-line Filters with Strongly-Coupled Resonator Triplet" IEEE Trans. Microw. Theory Techn., vol. 66, N. 12, pp. 5585-5592, Dec. 2018.

[16] M. Latif, G. Macchiarella and F. Mukhtar, "A Novel Coupling Structure for Inline Realization of Cross-Coupled Rectangular Waveguide Filters," in IEEE Access, vol. 8, pp. 107527-107538, 2020.

[17] T. Zhang, Z. Long, L. Zhou, M. Qiao, F. Hou and M. Tian, "Realization of Even Transmission Zeros for Filter Without Cross-Couplings," in IEEE Trans. Microw. Theory Tech., vol. 66, no. 12, pp. 5248-5259, Dec. 2018.

[18] S. Tamiazzo, G. Macchiarella and F. Seyfert, "A True Inline CoaxialCavity Filter with Two Symmetric Zeros," in IEEE Microw. Wireless Compon. Lett. vol. 31, no. 6, pp. 666-669, Jun. 2021.

[19] S. Amari, "On the maximum number of finite transmission zeros of coupled resonator filters with a given topology," IEEE Microw. Guided Wave Lett., vol. 9, no. 9, pp. 354-356, Sept. 1999.

[20] R. J. Cameron, C. M. Kudsia, and R. R. Mansour, Microwave Filters for Communication Systems. Hoboken, NJ, USA: John Wiley \& Sons, 2007

[21] D. Swanson, G. Macchiarella "Microwave filter design by synthesis and optimization" IEEE Microw. Mag., Vol. 8, n.2, pp. 52-69, May 2007. 


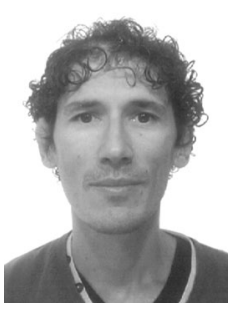

Stefano Tamiazzo was born in Milan, Italy on 1974. $\mathrm{He}$ graduated from Politecnico di Milano in telecommunication engineering in April 2001. He remained at Politecnico to complete a Master in Information Technology untill 2002. After receiving his education Stefano Tamiazzo worked as an RF passive engineer with Andrew Telecommunications in Agrate Brianza, Italy. His tasks included the design and testing of filters, duplexers and Tower Mounted Amplifiers (TMAs) for base stations of cellular wireless networks. He also supported the mass production of such devices focusing on PIM issues. $\mathrm{He}$ is co-author of several papers on filter synthesis and realization, most of them published on the IEEE Transactions. He is currently with Commscope Italy, which acquired Andrew in 2007. His current research interests include the development of miniaturized low-loss filters and combiners for base stations.

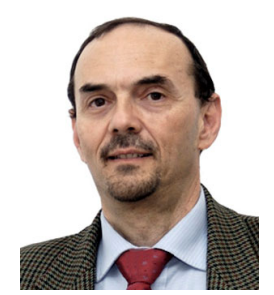

Giuseppe Macchiarella (M'90-SM'06-F15) is currently Professor of Microwave Engineering with the Department of Electronic, Information and Bioengineering, Politecnico di Milano, Italy. His research activity has covered in the past several areas of microwave engineering: microwave acoustics (SAW devices), radio wave propagation, numerical methods for electromagnetic, power amplifiers, linearization techniques. He has been Scientific Coordinator of PoliEri, a Research Laboratory on monolithic microwave integrated circuit (MMIC), which was jointly supported by Politecnico di Milano and Ericsson Company. Currently, his activities are focused on the development of new techniques for the synthesis of microwave filters and multiplexers. Prof. Macchiarella is Fellow of IEEE and authored or co-authored more than 160 papers on journals and conferences proceedings. He has been responsible of several contracts and collaborations with various Companies operating in the microwave industry. He has been Chair of IEEE Technical Committee MTT-8 (Filters and Passive Components). He is serving since several years in the TPC (Technical Program Committee) of IEEE International Microwave Symposium and European Microwave Conference.

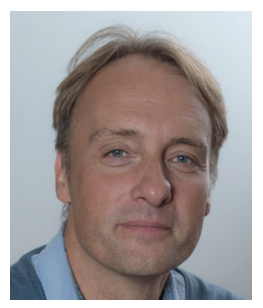

Fabien Seyfert received the Engineering degree from the Ecole Superieure des Mines de St. Etienne, St. Etienne, France, in 1993, and the Ph.D. degree in mathematics from the Ecole Superierure des Mines de Paris, Paris, France, in 1998. From 1998 to 2001, he was with Siemens, Munich, Germany, as a Researcher specializing in discrete and continuous optimization methods. Since 2002, he has been a Researcher with the Institut National de Recherche en Informatique et en Automatique (INRIA), SophiaAntipolis, France. His research interests are focused on the development of efficient mathematical procedures and associated software for signal processing including computer-aided techniques for the design and tuning of microwave devices. 